\title{
eHealth for Addressing Balance Disorders in the Elderly: Systematic Review
}

\author{
Andréa G Martins Gaspar ${ }^{1,2 *}, \mathrm{MD}$; Luís Velez Lapão $0^{2^{*}}, \mathrm{PhD}$ \\ ${ }^{1}$ Hospital Beatriz Ângelo, Lisbon, Portugal \\ ${ }^{2}$ Global Health and Tropical Medicine, Instituto de Higiene e Medicina Tropical, Universidade NOVA de Lisboa, Lisbon, Portugal \\ *all authors contributed equally
}

\section{Corresponding Author:}

Andréa G Martins Gaspar, MD

Global Health and Tropical Medicine

Instituto de Higiene e Medicina Tropical

Universidade NOVA de Lisboa

Rua da Junqueira, 100

Lisbon, 1349-008

Portugal

Phone: 351213652600

Email: andreamartinsbr@hotmail.com

\begin{abstract}
Background: The population is aging on a global scale, triggering vulnerability for chronic multimorbidity, balance disorders, and falls. Falls with injuries are the main cause of accidental death in the elderly population, representing a relevant public health problem. Balance disorder is a major risk factor for falling and represents one of the most frequent reasons for health care demand. The use of information and communication technologies to support distance healthcare (eHealth) represents an opportunity to improve the access and quality of health care services for the elderly. In recent years, several studies have addressed the potential of eHealth devices to assess the balance and risk of falling of elderly people. Remote rehabilitation has also been explored. However, the clinical applicability of these digital solutions for elderly people with balance disorders remains to be studied.
\end{abstract}

Objective: The aim of this review was to guide the clinical applicability of eHealth devices in providing the screening, assessment, and treatment of elderly people with balance disorders, but without neurological disease.

Methods: A systematic review was performed in accordance with the PRISMA (Preferred Reporting Items for Systematic Reviews and Meta-Analysis) statement. Data were obtained through searching the PubMed, Google Scholar, Embase, and SciELO databases. Only randomized controlled trials (RCTs) or quasiexperimental studies (QESs) published between January 2015 and December 2019 were included. The quality of the evidence to respond to the research question was assessed using Joanna Briggs Institute (JBI) Critical Appraisal for RCTs and the JBI Critical Appraisal Checklist for QESs. RCTs were assessed using the Cochrane risk of bias tool. We provide a narrative synthesis of the main outcomes from the included studies.

Results: Among 1030 unduplicated articles retrieved, 21 articles were included in this review. Twelve studies explored different technology devices to obtain data about balance and risk of falling. Nine studies focused on different types of balance exercise training. A wide range of clinical tests, functional scales, classifications of faller participants, sensor-based tasks, intervention protocols, and follow-up times were used. Only one study described the clinical conditions of the participants. Instrumental tests of the inner ear were neither used as the gold-standard test nor performed in pre and postrehabilitation assessments.

Conclusions: eHealth has potential for providing additional health care to elderly people with balance disorder and risk of falling. In the included literature, the heterogeneity of populations under study, methodologies, eHealth devices, and time of follow-up did not allow for clear comparison to guide proper clinical applicability. This suggests that more rigorous studies are needed.

(J Med Internet Res 2021;23(4):e22215) doi: 10.2196/22215

\section{KEYWORDS}

balance disorders; falls; elderly; eHealth; telemedicine 


\section{Introduction}

\section{Background}

\section{Aging and Balance Disorders}

The improvement of health conditions and the increase in life expectancy have led to an aging global population, although this is not always accompanied by an increase in healthy life years [1-4].

Aging is associated with functional deterioration, including in the peripheral sensory structures, thereby affecting vision, hearing, and balance [5,6]. Additionally, elderly individuals are more likely to suffer from multiple chronic conditions, which often leads to frailty with risk of falls [1-4]. Falls in elderly people represent a serious public health problem as the main cause of accidental death in this population. The risk of falling increases with age [1,7-9]. Each year, approximately one in every three elderly people experiences a serious fall. Moreover, falling can lead to deterioration of the quality of life, anxiety, depression, restriction in daily activities, decreased mobility, social isolation, increased consumption of medications, and increased dependence on medical services and informal caregivers [1,2].

Several causes of falls in the elderly population have been identified, including age, environmental factors (eg, wet paths), inappropriate clothing and shoes, incorrect behavior (eg, climbing chairs), excessive alcohol consumption, inadequate use of medications, deteriorating chronic illness, and balance disorders $[1,6,10]$.

Various clinical conditions are associated with balance disorders in elderly people, including age-related decline in balance function (prebyvestibulopathy); medications; and cardiovascular, metabolic, musculoskeletal, neurologic, and otologic diseases $[5,6]$.

Although dizziness and vertigo are recognized as significant factors increasing the risk of falling and are common symptoms among the elderly, epidemiological studies have revealed large variability in the prevalence of balance disorders in this population [11-13]. It is estimated that at least $30 \%$ of individuals above 60 years old suffer from vertigo and dizziness, increasing to $50 \%$ for those above 85 years old [13]. According to the 2008 National Health Interview Survey, 33 million US adults had balance disorders, $26 \%$ of whom were elderly people (above 65 years) [14]. Approximately $20 \%$ of elderly people in the United States have a balance disorder event annually [15]. In fact, dizziness is a common complaint among the elderly population and is a strong predictor of falling events with a negative impact on quality of life [16]. Poor balance is frequently associated with falling [17,18]. In particular, asymmetrical vestibular function may often contribute to falls and fractures in elderly people [19-21].

Balance disorders and consequent falls have progressively represented a burden of disease, accompanied by high costs and pressure on the social services and health care systems related to medical care. This includes repeated consultations, excessive use of diagnostic imaging, and emergency care [22-24]. For example, the first national study in the context of dizziness and vertigo in the Emergency Services of United States of America for 2011 revealed that $25.7 \%$ of patient complaints of dizziness and vertigo were associated with balance disorders. The cost was estimated at about US $\$ 768$ per episode, translating to an annual national cost of US $\$ 757$ million. In the same context, cardiovascular diseases (linked to $16.5 \%$ of these episodes) represented a cost of approximately US $\$ 1489$ per episode for an annual cost of US $\$ 941$ million. By comparison, cerebrovascular diseases only accounted for $3.1 \%$ of these episodes, but with a cost per episode of approximately US \$1059 or an annual cost of US $\$ 127$ million. With the progressive aging of the population, worsening of this situation is expected in the future [25]. Indeed, vertigo is already contributing to the increasing trend of health care costs, which is linked to the aging of the population $[23,24]$.

In this scenario of global aging, the use of digital solutions has been encouraged. Moreover, the additional pressure of the current COVID-19 pandemic has motivated the broader use of eHealth technologies [26].

\section{Digital Health Care and the Elderly}

The aging trend represents a relevant challenge to both patients and their families, and to the sustainability of health care systems globally. This is linked to the goal of global health policies for achieving a more active and healthy aging society with autonomy and independence [27,28]. The provision of new health care models, including eHealth services, has been encouraged to tackle access inequities, optimize health outcomes, and ensure autonomy and social support for elderly people. The use of eHealth seems to decrease costs associated with both institutionalization and unnecessary hospital visits [27-29].

eHealth consists of the use of information and communication technologies (ICTs) to support a health care communication channel at a distance, allowing for more efficient delivery of care services with optimized resource allocation. eHealth often contributes to improving the quality of health care services, including faster access to health information, promotion of the globalization of health care, and better health outcomes [30]. The World Health Organization has also recommended eHealth to promote universal health coverage, envisaging higher health care services availability with fewer resources and larger patient interaction. To date, eHealth has been used in the management of many conditions from health literacy promotion to teleconsultations [31]. The remote access systems can actively monitor elderly people in a real-life environment, leveraging the fact that there is an increasing interest and engagement of the elderly with technology. Moreover, eHealth technologies can enhance medical-patient interactions and mitigate many care access inequities. However, digital training of elderly people and caregivers is essential [4,32-34].

\section{Assessment and Rehabilitation for the Elderly with Balance Disorders}

There are several clinical tests and functional scales, including the Timed Up and Go Test (TUGT), Unipedal Standing Test, and Berg Balance Scale, that allow for assessments of balance, 
gait, and risk of falling [5,35]. The use of sensors can improve the data quality of these tests and scales [36,37]. Additionally, functional tests of the inner ear, such as videonystagmography or the Video Head Impulse Test, are essential to identify and measure balance disorder cases, including an age-related decline in balance function (prebyvestibulopathy) [6].

Personalized balance training is a relevant option for the treatment of elderly people with balance disorders and risk of falling [5]. This training consists of an exercise-based program to address an individual's specific balance disorder, with goals of increasing postural stability, improving activities of daily living, and decreasing symptoms. Balance training should be focused on the functional deficiencies identified. Therefore, a prior medical evaluation is necessary to identify the clinical conditions related to poor balance as mentioned previously [38-41]. Moreover, these clinical conditions can affect the outcomes. For example, intervention success is more difficult when the patient has a disorder of both inner ears or has limited mobility due to an osteoarticular disease [38-41]. Exercises delivered through video games can be a promising intervention to achieve greater access and adherence among elderly people $[42,43]$.

Several reviews have addressed the potential of digital solutions to improve the clinical observation and evaluation of balance disorders, and to promote the remote balance rehabilitation of elderly people [36,37,42-47]. However, most of these reviews included studies using a younger population as a preliminary assessment [42,44-47], and the majority did not describe the clinical conditions of the participants that might interfere with the outcomes, especially in the context of balance rehabilitation. Additionally, the clinical applicability of these devices was not assessed [36,37,42-47].
Therefore, there is a gap in this field in terms of evaluating the overall applicability of digital solutions according to the clinical conditions of elderly people with balance disorders and without neurological disease.

\section{Objectives}

The aim of this review was to evaluate and guide the clinical applicability of eHealth devices in the screening, assessment, and treatment of elderly people with balance disorders but without neurological disease.

\section{Methods}

\section{Design}

This systematic review was performed in accordance with the PRISMA (Preferred Reporting Items for Systematic Reviews and Meta-Analysis) statement [48] with the following steps: development of research questions, development of a search strategy with eligibility criteria, data selection, and qualitative analysis.

The protocol for this systematic review was registered in the International Prospective Register of Systematic Reviews (PROSPERO; CRD42019120774) and the complete protocol is available on the National Institute for Health Research program website.

This review focused on answering the following specific research questions, according to the PICO (Population, patient, or problem; Intervention; Control, Comparison, or Comparator; Outcome) strategy [49] (Textbox 1): (1) What are the main contributions of eHealth to elderly people with balance disorders with risk of falling? and (2) Is there any evidence that eHealth improves the quality of health care services in this context? If not, what are the reasons?

Textbox 1. Description of the PICO components.

- P (Population, Patient, Problem): Elderly people (over 60 years old) with balance disorders and risk of falling; studies with elderly people with functional limitation by neurological disease were excluded

- I Intervention): eHealth devices for remote health education, screening, assessment, monitoring, or rehabilitation of elderly people with balance disorders with risk of falling

- $\quad \mathrm{C}$ (Control, Comparison, Comparator): No intervention, paper booklet information, clinical evaluation, conservative balance training

- O (Outcome): Clinical applicability, increased fall prevention literacy, early identification and evaluation of balance deficits and risk of falling, improved balance and gait performance, reduced rate of falling, increased rehabilitation adherence, increased independence in daily activities

\section{Definition of Concepts and Keywords used in the Search Strategy}

In this study, we defined elderly people as those over 60 years of age [50]. Knudson [51] defined balance as a "person's ability to control their body position relative to some base of support." According to Agrawal et al [6], vertigo and dizziness are defined as "sensation of self-motion when no self-motion is occurring or the sensation of distorted self-motion during an otherwise normal head movement" and "sensation of disturbed or impaired spatial orientation without a false or distorted sense of motion," respectively. Falls refer to "inadvertently landing on the ground, floor or other lower level" [10]. Gait is defined as "the pattern of movement of the body during locomotion" [52].
Telemedicine is defined according to the World Health Organization Group Consultation on Health Telematics [53] as "delivery of health care services using ICT for the exchange of valid information for diagnosis, treatment and prevention of disease and injuries, research and evaluation, and for the continuing education of health care providers." eHealth is defined according to Eysenbach [54] as:

an emerging field in the intersection of medical informatics, public health and business, referring to health services and information delivered or enhanced through the Internet and related technologies. In a broader sense, the term characterizes not only a technical development, but also a state-of-mind, a 
way of thinking, an attitude, and a commitment for networked, global thinking, to improve health care locally, regionally, and worldwide by using information and communication technology.

Teleconsultation is defined as "synchronous or asynchronous consultation using ICT to omit geographical and functional distance" [55]. Finally, a sensor is defined as a "device that responds to a physical input of interest with a recordable functionally related output that is usually electrical or optical" [56].

\section{Search Strategy}

Articles were retrieved through searching the PubMed, Google Scholar, Embase, and SciELO databases. The search algorithm included multiple group combinations, as shown in Table 1.

Table 1. Search strategy.

\begin{tabular}{|c|c|}
\hline Concept & Keywords \\
\hline Elderly people & (“elderly” OR “older” OR “aged”) \\
\hline Balance & $\begin{array}{l}\text { ("Balance" OR "balance disorder" OR "balance problem" OR "vertigo" OR "dizziness) and/or ("falls" OR "fall } \\
\text { detection" OR "fall prevention") and/or ("gait") }\end{array}$ \\
\hline Telemedicine & (“Telemedicine” OR “eHealth” OR “teleconsultation” OR “technology” OR “sensor”) \\
\hline
\end{tabular}

\section{Selection Criteria}

The inclusion criteria were randomized controlled trials (RCTs) or quasiexperimental studies (QESs) published in English between January 2015 and December 2019, studies related to use of eHealth in the context of balance and falls, and the sample was restricted to an elderly population (60 years old and above).

The exclusion criteria were: (1) review articles, brief reports, protocols, proof-of-concepts, pilot studies, conference papers, and letters to the editor; (2) studies including elderly people with a reported functional limitation due to a neurological disease; and (3) articles without an age sample reference or with participants aged below 60 years.

\section{Screening Process and Data Extraction}

First, both authors screened the papers independently, looking at titles, abstracts, and methods, and agreed about their inclusion or exclusion according to the eligibility criteria. Second, the potentially relevant papers were retrieved for full-text evaluation against the eligibility criteria. Any articles that were deemed to be questionable in the first stage were included for further evaluation in the second stage. The selection of papers was performed by checking the extracted data and risk of bias.

\section{Outcome Measures}

The main outcomes included population characteristics, balance disorder, identification of faller participants, eHealth platform and services, health benefits, and fall prevention literacy.

\section{Risk of Bias Assessment}

The quality of the evidence to respond to the research questions was independently assessed using the Joanna Briggs Institute
(JBI) Critical Appraisal for Experimental Studies and JBI Critical Appraisal Checklist for Quasi-Experimental Studies tools [57]. The two researchers discussed the results of the quality appraisal, reaching a consensus in case of any divergence. The included RCTs were assessed using the Cochrane risk of bias tool [58] to evaluate the risk of internal bias for a series of domains: selection bias, performance bias, detection bias, attrition bias, and reporting bias. Disagreements were solved by consensus between the two researchers.

\section{Data Analysis}

We provide a narrative synthesis of the main outcomes from the included studies. First, the articles were categorized according to the study design. Second, the articles were categorized based on the focus of eHealth services (screening/assessment and treatment/rehabilitation) for comparison of clinical use and applicability according to digital devices.

\section{Results}

\section{Search Results}

A total of 1030 unduplicated articles were identified, 984 of which were excluded after title and abstract screening. Among the 46 full-text publications assessed for eligibility, 25 articles were excluded owing to functional limitations due to neurological disease $(n=4)$, age of participants $(n=5)$, focus on technological implementation $(n=2)$ or model/algorithm $(n=8)$, and specific descriptions of elderly gait parameters $(n=6)$.

Twenty-one articles [59-79] were ultimately included in the review (Figure 1). 
Figure 1. Flow of selection for studies included and excluded in the review.

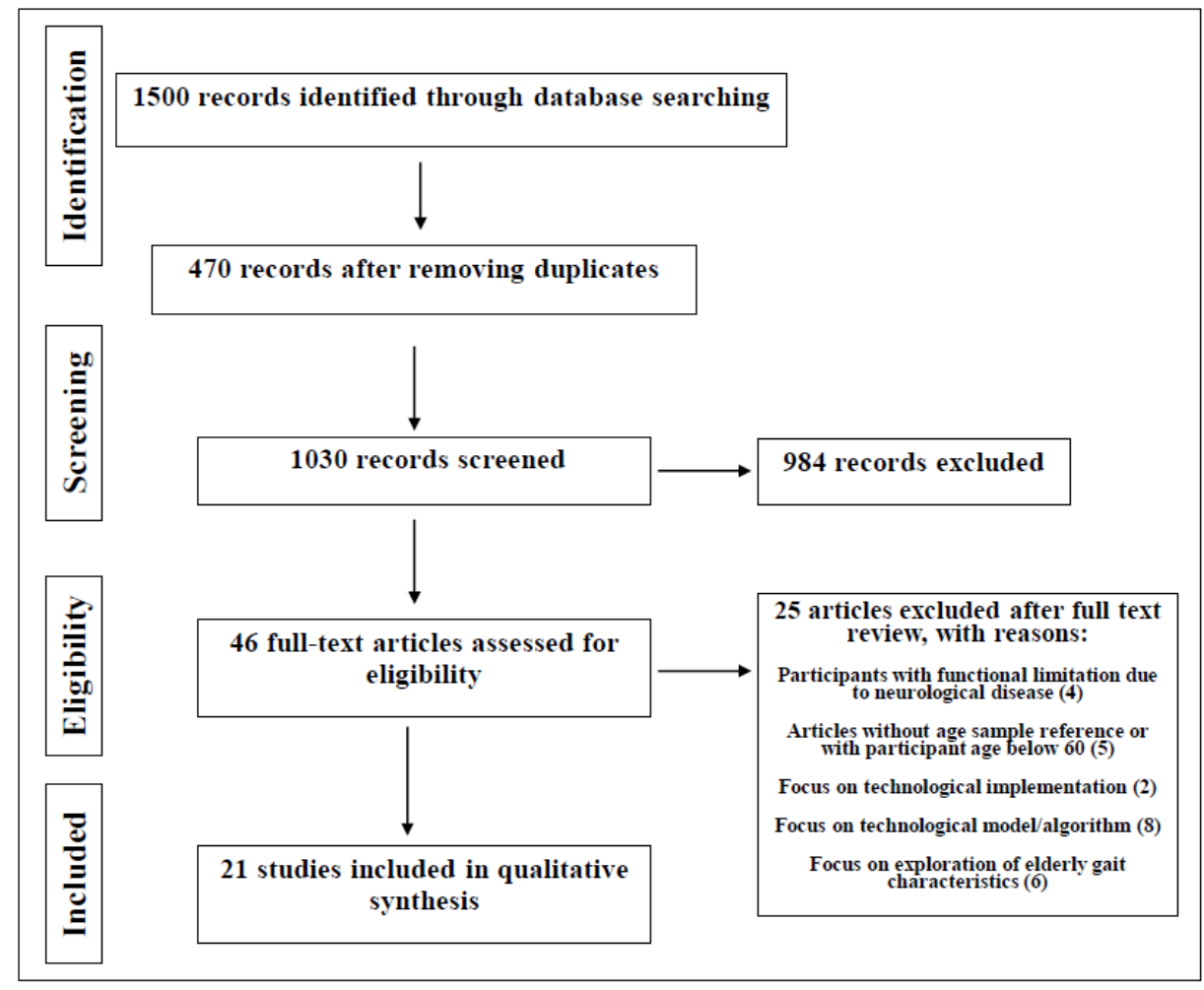

\section{Study Design}

\section{RCT Design}

Seven studies were RCTs [59-65], including one cross-over trial without a washout term [64] and one multicenter study [60].

Not all authors clearly described the randomization process [62] and the allocation concealment [62,64] (Tables 2 and 3).

The inclusion criteria were mentioned in all articles. However, the clinical conditions of the participants were only described in one study [60]. The function of the inner ear was never mentioned. Thus, the expected similarity between the control and intervention groups was not clear. This is relevant because various clinical conditions (eg, cardiovascular, metabolic, inner ear disease, medication) can interfere with the outcomes of balance rehabilitation [38-41]. Therefore, the lack of information about clinical conditions of the participants, including the lack of data about function of the inner ear, was considered as "other bias" and was a common weakness of all included RCTs (Table 3 and Figure 2). This approach led to a worse classification of the quality of these studies.

Additionally, the blinding of participants, personnel, and outcome assessment were unclear in some of these studies.

In the control and intervention groups of all RCTs, a few dropouts for medical and personal reasons were mentioned. However, this was not considered to be sufficiently relevant to have an impact on the results. Only two papers reported intention-to-treat analysis [60,63].

All outcomes were measured in a reliable manner and were considered to have been properly analyzed. 
Table 2. Methodological quality of randomized controlled trials based on the Joanna Briggs Institute Critical Appraisal Checklist.

\begin{tabular}{|c|c|c|c|c|c|c|c|c|c|c|c|c|c|}
\hline Study & $\mathrm{Q} 1^{\mathrm{a}}$ & $\mathrm{Q}^{2}{ }^{\mathrm{b}}$ & $\mathrm{Q}^{\mathrm{c}}$ & $\mathrm{Q} 4^{\mathrm{d}}$ & $\mathrm{Q} 5^{\mathrm{e}}$ & $\mathrm{Q} 6^{\mathrm{f}}$ & $\mathrm{Q}^{\mathrm{g}}$ & $\mathrm{Q}^{\mathrm{h}}$ & $\mathrm{Q}^{\mathrm{i}}$ & Q10 & $\mathrm{Q} 11^{\mathrm{k}}$ & $\mathrm{Q}^{1} 2^{1}$ & $\mathrm{Q} 13^{\mathrm{m}}$ \\
\hline $\begin{array}{l}\text { Eggenberger et al } \\
\text { [59] }\end{array}$ & $\mathrm{Y}^{\mathrm{n}}$ & $\mathrm{Y}$ & $\mathrm{U}^{\mathrm{o}}$ & $\mathrm{Y}$ & $\mathrm{N}^{\mathrm{p}}$ & $\mathrm{U}$ & $\mathrm{Y}$ & $\mathrm{Y}$ & $\mathrm{N}$ & $\mathrm{Y}$ & $\mathrm{Y}$ & $\mathrm{Y}$ & $\mathrm{Y}$ \\
\hline Gschwind et al [60] & $\mathrm{Y}$ & $\mathrm{Y}$ & $\mathrm{U}$ & $\mathrm{Y}$ & $\mathrm{Y}$ & $\mathrm{U}$ & $\mathrm{Y}$ & $\mathrm{Y}$ & $\mathrm{Y}$ & $\mathrm{Y}$ & $\mathrm{Y}$ & $\mathrm{Y}$ & $\mathrm{Y}$ \\
\hline Gschwind et al [61] & $\mathrm{Y}$ & $\mathrm{Y}$ & $\mathrm{U}$ & $\mathrm{U}$ & $\mathrm{U}$ & $\mathrm{Y}$ & $\mathrm{Y}$ & $\mathrm{Y}$ & $\mathrm{N}$ & $\mathrm{Y}$ & $\mathrm{Y}$ & $\mathrm{Y}$ & $\mathrm{Y}$ \\
\hline Lim et al [62] & $\mathrm{U}$ & $\mathrm{U}$ & $\mathrm{U}$ & $\mathrm{U}$ & $\mathrm{U}$ & $\mathrm{U}$ & $\mathrm{Y}$ & $\mathrm{Y}$ & $\mathrm{N}$ & $\mathrm{Y}$ & $\mathrm{Y}$ & $\mathrm{Y}$ & $\mathrm{Y}$ \\
\hline Oesch et al [63] & $\mathrm{Y}$ & $\mathrm{Y}$ & $\mathrm{U}$ & $\mathrm{N}$ & $\mathrm{N}$ & $\mathrm{Y}$ & $\mathrm{Y}$ & $\mathrm{Y}$ & $\mathrm{Y}$ & $\mathrm{Y}$ & $\mathrm{Y}$ & $\mathrm{Y}$ & $\mathrm{Y}$ \\
\hline Ozaki et al [64] & $\mathrm{Y}$ & $\mathrm{U}$ & $\mathrm{U}$ & $\mathrm{U}$ & $\mathrm{U}$ & $\mathrm{U}$ & $\mathrm{Y}$ & $\mathrm{Y}$ & $\mathrm{N}$ & $\mathrm{Y}$ & $\mathrm{Y}$ & $\mathrm{Y}$ & $\mathrm{Y}$ \\
\hline Hong et al [65] & $\mathrm{Y}$ & $\mathrm{Y}$ & $\mathrm{U}$ & $\mathrm{Y}$ & $\mathrm{Y}$ & $\mathrm{Y}$ & $\mathrm{Y}$ & $\mathrm{Y}$ & $\mathrm{N}$ & $\mathrm{Y}$ & $\mathrm{Y}$ & $\mathrm{Y}$ & $\mathrm{Y}$ \\
\hline
\end{tabular}

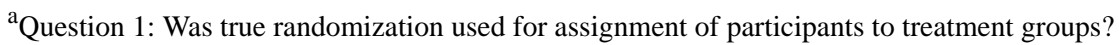

${ }^{\mathrm{b}}$ Question 2: Was allocation to treatment groups concealed?

${ }^{\mathrm{c}}$ Question 3: Were treatment groups similar at the baseline?

${ }^{\mathrm{d}}$ Question 4: Were participants blind to treatment assignment?

${ }^{\mathrm{e}}$ Question 5: Were those delivering treatment blind to treatment assignment?

${ }^{\mathrm{f}}$ Question 6: Were outcomes assessors blind to treatment assignment?

${ }^{\mathrm{g}}$ Question 7: Were treatment groups treated identically other than the intervention of interest?

${ }^{\mathrm{h}}$ Question 8: Was follow-up complete and if not, were differences between groups in terms of their follow-up adequately described and analyzed?

${ }^{\mathrm{i}}$ Question 9: Were participants analyzed in the groups to which they were randomized?

${ }^{\mathrm{j}}$ Question 10: Were outcomes measured in the same way for treatment groups?

${ }^{\mathrm{k}}$ Question 11: Were outcomes measured in a reliable way?

${ }^{1}$ Question 12: Was appropriate statistical analysis used?

${ }^{\mathrm{m}}$ Question 13: Was the trial design appropriate, and any deviations from the standard randomized controlled trial design (individual randomization, parallel groups) accounted for in the conduct and analysis of the trial?

${ }^{\mathrm{n}} \mathrm{Y}$ : Yes.

${ }^{\mathrm{o}} \mathrm{N}$ : No.

pU: Unclear.

Table 3. Risk of bias for randomized controlled trials based on the modified Cochrane Collaboration tool.

\begin{tabular}{|c|c|c|c|c|c|c|c|}
\hline \multirow[t]{2}{*}{ Study } & \multicolumn{2}{|l|}{ Selection bias } & $\begin{array}{l}\text { Other } \\
\text { bias }\end{array}$ & $\begin{array}{l}\text { Reporting } \\
\text { bias: selective } \\
\text { reporting }\end{array}$ & $\begin{array}{l}\text { Performance bias: } \\
\text { blinding (partici- } \\
\text { pants and personnel) }\end{array}$ & $\begin{array}{l}\text { Detection bias: } \\
\text { blinding (outcome } \\
\text { assessment) }\end{array}$ & $\begin{array}{l}\text { Attrition bias: } \\
\text { incomplete out- } \\
\text { come data }\end{array}$ \\
\hline & $\begin{array}{l}\text { Random sequence } \\
\text { generation }\end{array}$ & $\begin{array}{l}\text { Allocation } \\
\text { concealment }\end{array}$ & & & & & \\
\hline $\begin{array}{l}\text { Eggenberger et al } \\
{[59]}\end{array}$ & $\mathrm{L}^{\mathrm{a}}$ & $\mathrm{L}$ & $\mathrm{H}^{\mathrm{b}}$ & $\mathrm{U}^{\mathrm{c}}$ & $\mathrm{L}$ & $\mathrm{U}$ & $\mathrm{L}$ \\
\hline Gschwind et al [60] & $\mathrm{L}$ & $\mathrm{L}$ & $\mathrm{H}$ & $\mathrm{L}$ & $\mathrm{L}$ & $\mathrm{U}$ & $\mathrm{L}$ \\
\hline Gschwind et al [61] & $\mathrm{L}$ & $\mathrm{L}$ & $\mathrm{H}$ & $\mathrm{L}$ & $\mathrm{U}$ & $\mathrm{L}$ & $\mathrm{L}$ \\
\hline Lim et al [62] & $\mathrm{U}$ & $\mathrm{U}$ & $\mathrm{H}$ & $\mathrm{L}$ & $\mathrm{U}$ & $\mathrm{U}$ & $\mathrm{L}$ \\
\hline Oesch et al [63] & $\mathrm{L}$ & $\mathrm{L}$ & $\mathrm{H}$ & $\mathrm{L}$ & $\mathrm{H}$ & $\mathrm{L}$ & $\mathrm{L}$ \\
\hline Ozaki et al [64] & $\mathrm{L}$ & $\mathrm{U}$ & $\mathrm{H}$ & $\mathrm{L}$ & $\mathrm{U}$ & $\mathrm{U}$ & $\mathrm{L}$ \\
\hline
\end{tabular}

${ }^{\mathrm{a}} \mathrm{L}$ : low risk.

${ }^{\mathrm{b}} \mathrm{H}$ : high risk.

${ }^{\mathrm{c}} \mathrm{U}$ : unclear risk. 
Figure 2. Risk of bias in accordance with the authors' judgment (RevMan version 5.3.).

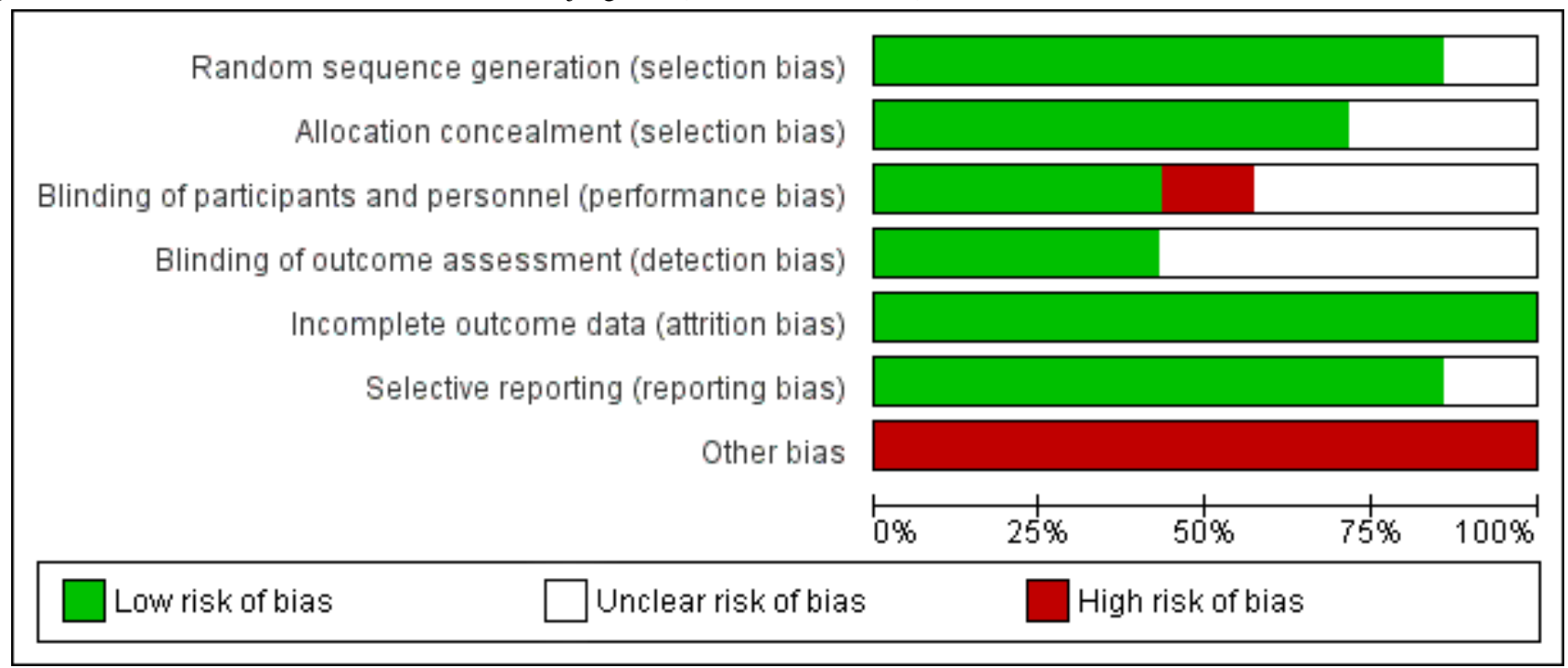

\section{QES Design}

Fourteen studies were QESs [66-79]; only one of these was a multicenter study [73]. Twelve of these studies used the same group of participants $[66,68-77,79]$. One study used two groups with different participants [67] and another had a control group and an intervention group [78]. However, the expected similarity between the groups was not clear because there was no description of the clinical conditions of the participants, including function of the inner ear. Loss to follow-up was also not mentioned for any of these studies (Table 4). 
Table 4. Methodological quality of quasiexperimental studies based on the Joanna Briggs Institute Critical Appraisal Checklist.

\begin{tabular}{|c|c|c|c|c|c|c|c|c|c|}
\hline Study & $\mathrm{Q}^{\mathrm{a}}$ & $\mathrm{Q} 2^{\mathrm{b}}$ & $\mathrm{Q}^{\mathrm{c}}$ & $\mathrm{Q} 4^{\mathrm{d}}$ & $\mathrm{Q} 5^{\mathrm{e}}$ & $\mathrm{Q} 6^{\mathrm{f}}$ & $\mathrm{Q}^{\mathrm{g}}$ & Q8 ${ }^{\mathrm{h}}$ & $\mathrm{Q}^{\mathrm{i}}$ \\
\hline Zacaria et al [66] & $\mathrm{Y}^{\mathrm{j}}$ & $\mathrm{Y}$ & $\mathrm{Y}$ & $\mathrm{N}^{\mathrm{k}}$ & $\mathrm{Y}$ & $\mathrm{Y}$ & $\mathrm{Y}$ & $\mathrm{Y}$ & $\mathrm{Y}$ \\
\hline Hall et al [67] & $\mathrm{Y}$ & $\mathrm{U}^{1}$ & $\mathrm{Y}$ & $\mathrm{N}$ & $\mathrm{Y}$ & $\mathrm{Y}$ & $\mathrm{Y}$ & $\mathrm{Y}$ & $\mathrm{Y}$ \\
\hline Howcroft et al [68] & $\mathrm{Y}$ & $\mathrm{Y}$ & $\mathrm{Y}$ & $\mathrm{N}$ & $\mathrm{Y}$ & $\mathrm{Y}$ & $\mathrm{Y}$ & $\mathrm{Y}$ & $\mathrm{Y}$ \\
\hline Lee et al [69] & $\mathrm{Y}$ & $\mathrm{Y}$ & $\mathrm{Y}$ & $\mathrm{N}$ & $\mathrm{Y}$ & $\mathrm{Y}$ & $\mathrm{Y}$ & $\mathrm{Y}$ & $\mathrm{Y}$ \\
\hline Ponti et al [70] & $\mathrm{Y}$ & $\mathrm{Y}$ & $\mathrm{Y}$ & $\mathrm{N}$ & $\mathrm{Y}$ & $\mathrm{Y}$ & $\mathrm{Y}$ & $\mathrm{Y}$ & $\mathrm{Y}$ \\
\hline Similä et al [71] & $\mathrm{Y}$ & $\mathrm{Y}$ & $\mathrm{Y}$ & $\mathrm{N}$ & $\mathrm{Y}$ & $\mathrm{Y}$ & $\mathrm{Y}$ & $\mathrm{Y}$ & $\mathrm{Y}$ \\
\hline Shahzad et al [72] & $\mathrm{Y}$ & $\mathrm{Y}$ & $\mathrm{Y}$ & $\mathrm{N}$ & $\mathrm{Y}$ & $\mathrm{Y}$ & $\mathrm{Y}$ & $\mathrm{Y}$ & $\mathrm{Y}$ \\
\hline Brodie et al [73] & $\mathrm{Y}$ & $\mathrm{Y}$ & $\mathrm{Y}$ & $\mathrm{N}$ & $\mathrm{Y}$ & $\mathrm{Y}$ & $\mathrm{Y}$ & $\mathrm{Y}$ & $\mathrm{Y}$ \\
\hline Howcroft et al [74] & $\mathrm{Y}$ & $\mathrm{Y}$ & $\mathrm{Y}$ & $\mathrm{N}$ & $\mathrm{Y}$ & $\mathrm{Y}$ & $\mathrm{Y}$ & $\mathrm{Y}$ & $\mathrm{Y}$ \\
\hline Chigateri et al [75] & $\mathrm{Y}$ & $\mathrm{Y}$ & $\mathrm{Y}$ & $\mathrm{N}$ & $\mathrm{Y}$ & $\mathrm{Y}$ & $\mathrm{Y}$ & $\mathrm{Y}$ & $\mathrm{Y}$ \\
\hline Qiu et al [76] & $\mathrm{Y}$ & $\mathrm{Y}$ & $\mathrm{Y}$ & $\mathrm{N}$ & $\mathrm{Y}$ & $\mathrm{Y}$ & $\mathrm{Y}$ & $\mathrm{Y}$ & $\mathrm{Y}$ \\
\hline Hiesh et al [77] & $\mathrm{Y}$ & $\mathrm{Y}$ & $\mathrm{Y}$ & $\mathrm{N}$ & $\mathrm{Y}$ & $\mathrm{Y}$ & $\mathrm{Y}$ & $\mathrm{Y}$ & $\mathrm{Y}$ \\
\hline Maneproom et al [78] & $\mathrm{Y}$ & $\mathrm{U}$ & $\mathrm{Y}$ & $\mathrm{Y}$ & $\mathrm{Y}$ & $\mathrm{Y}$ & $\mathrm{Y}$ & $\mathrm{Y}$ & $\mathrm{Y}$ \\
\hline Nightingale et al [79] & $\mathrm{Y}$ & $\mathrm{Y}$ & $\mathrm{Y}$ & $\mathrm{N}$ & $\mathrm{Y}$ & $\mathrm{Y}$ & $\mathrm{Y}$ & $\mathrm{Y}$ & $\mathrm{Y}$ \\
\hline
\end{tabular}

aQuestion 1: Is it clear in the study what is the "cause" and what is the "effect" (ie, there is no confusion about which variable comes first)?

${ }^{\mathrm{b}}$ Question 2: Were the participants included in any similar comparisons?

${ }^{\mathrm{c}}$ Question 3: Were the participants included in any comparisons receiving similar treatment/care other than the exposure or intervention of interest?

${ }^{\mathrm{d}}$ Question 4: Was there a control group?

${ }^{\mathrm{e}}$ Question 5: Were there multiple measurements of the outcome, both pre and post the intervention/exposure?

${ }^{\mathrm{f}}$ Question 6: Was follow-up completed and if not, were differences between groups in terms of their follow-up adequately described and analyzed?

${ }^{\mathrm{g}}$ Question 7: Were the outcomes of participants included in any comparisons measured in the same way?

${ }^{\mathrm{h}}$ Question 8: Were outcomes measured in a reliable way?

${ }^{\mathrm{i}}$ Question 9: Was appropriate statistical analysis used?

${ }^{\mathrm{j}} \mathrm{Y}$ : yes.

$\mathrm{k}_{\mathrm{N}}$ : no.

${ }^{1} \mathrm{U}$ : unclear.

Multiple different measurements of the outcomes were used (Table 4). However, the instrumental inner ear tests were not used as the gold-standard test. This lack of comparison was considered to be a weakness of all of the included QESs. The most commonly applied tests were the TUGT and walking over different distances. One study assessed 1-week daily-life walking [73]. Only one study explored the activities of daily living [75] (Table 5).

The outcomes were considered to have been measured in a reliable manner and were properly analyzed. 
Table 5. Quasiexperimental studies focused on screening/assessment.

\begin{tabular}{|c|c|c|c|c|c|}
\hline Reference & $\begin{array}{l}\text { Population and set- } \\
\text { ting }\end{array}$ & $\begin{array}{l}\text { Participants, N } \\
\text { (male/female) }\end{array}$ & $\begin{array}{l}\text { Technology/sensor } \\
\text { used (location) }\end{array}$ & Completed sensor-based procedures & $\begin{array}{l}\text { Comparison with fall history } \\
\text { or a gold-standard test }\end{array}$ \\
\hline $\begin{array}{l}\text { Zacaria et al } \\
{[66]}\end{array}$ & Hospital & $38(20 / 18)$ & $\begin{array}{l}\text { WIS }{ }^{\mathrm{a}} \text { accelerometer } \\
\text { and gyrosensor (L2 } \\
\text { vertebra) }\end{array}$ & $\begin{array}{l}\text { TUGT }^{\mathrm{b}} \text { (sensor analysis of perfor- } \\
\text { mance of each phase) }\end{array}$ & $\begin{array}{l}\text { Classification of faller/nonfall- } \\
\text { er based on total duration for } \\
\text { TUGT completion }\end{array}$ \\
\hline $\begin{array}{l}\text { Howcroft et al } \\
{[68]}\end{array}$ & $\mathrm{CDEP}^{\mathrm{c}}$ & $100(44 / 56)$ & $\begin{array}{l}\text { Wearable pressure- } \\
\text { sensing insoles (pres- } \\
\text { sure sensors-plantar); } \\
\text { WIS, } 4 \text { triaxial ac- } \\
\text { celerometers (head, } \\
\text { pelvis, shanks) }\end{array}$ & $\begin{array}{l}7.62 \mathrm{~m} \text { walk and } 7.62 \mathrm{~m} \text { walk with } \\
\text { cognitive load (ST }{ }^{\mathrm{d}} \text { and } \mathrm{DT}^{\mathrm{e}} \text { gait) }\end{array}$ & $\begin{array}{l}\text { Classification of faller/nonfall- } \\
\text { er based on retrospective fall } \\
\text { occurrence }\end{array}$ \\
\hline Lee et al [69] & CDEP & $65(16 / 49)$ & $\begin{array}{l}\text { WIS (1 triaxial ac- } \\
\text { celerometer belt } \\
\text { around waist, pelvis, } \\
\text { sacrum, L3-L5 verte- } \\
\text { brae) }\end{array}$ & TUGT & $\begin{array}{l}\text { Short-form BBS }{ }^{\mathrm{f}} \text { ( } 7 \text { activi- } \\
\text { ties), TUGT }\end{array}$ \\
\hline Ponti et al [70] & CDEP & $36(11 / 25)$ & $\begin{array}{l}\text { WIS ( } 1 \text { triaxial ac- } \\
\text { celerometer, waist) }\end{array}$ & $\begin{array}{l}\text { ST TUGT, DT manual TUGT, DT } \\
\text { cognitive TUGT }\end{array}$ & $\begin{array}{l}\text { Faller/nonfaller based on ret- } \\
\text { rospective fall occurrence, } \\
\text { FES }^{\mathrm{g}}\end{array}$ \\
\hline Similä et al [71] & $\begin{array}{l}\text { Senior house/se- } \\
\text { nior physical exer- } \\
\text { cise group }\end{array}$ & $35(0 / 35)$ & $\begin{array}{l}\text { WIS ( } 2 \text { accelerome- } \\
\text { ters L3-L5 vertebrae, } \\
\text { right hip) }\end{array}$ & $\begin{array}{l}\text { BBS + TUGT + } 4 \mathrm{~m} \text { walk and fol- } \\
\text { low-up after } 1 \text { year }\end{array}$ & $\begin{array}{l}\text { Background questionnaire, } \\
\text { interview, balance platform } \\
\text { assessment with Kinect } \\
\text { recording }\end{array}$ \\
\hline $\begin{array}{l}\text { Shahzad et al } \\
{[72]}\end{array}$ & CDEP & $23(7 / 16)$ & $\begin{array}{l}\text { WIS ( } 1 \text { triaxial ac- } \\
\text { celerometer, L3-L5 } \\
\text { vertebrae) }\end{array}$ & TUGT, STS-5 ${ }^{\mathrm{h}}, \mathrm{AST}^{\mathrm{i}}$ & BBS \\
\hline $\begin{array}{l}\text { Brodie et al } \\
\text { [73] }\end{array}$ & CDEP & $96(39 / 57)$ & $\begin{array}{l}\text { Pendant sensor 3D ac- } \\
\text { celerometer and } \\
\text { barometer (sternum) }\end{array}$ & 1-week daily life walking & $\begin{array}{l}\text { Fall history, comparison with } \\
\text { TUGT and } 10 \mathrm{~m} \text { walk test }\end{array}$ \\
\hline $\begin{array}{l}\text { Howcroft et al } \\
{[74]}\end{array}$ & CDEP & $75(31 / 44)$ & $\begin{array}{l}\text { Wearable pressure- } \\
\text { sensing insoles (pres- } \\
\text { sure sensor, plantar), } \\
\text { WIS } 4 \text { triaxial ac- } \\
\text { celerometers (head, } \\
\text { pelvis, shanks) }\end{array}$ & $\begin{array}{l}7.62 \mathrm{~m} \text { walk under ST, } 7.62 \mathrm{~m} \text { walk } \\
\text { under DT (verbal-task cognitive } \\
\text { load) }\end{array}$ & $\begin{array}{l}\text { Classification of faller/ non- } \\
\text { faller based on prospective } \\
\text { fall occurrence }\end{array}$ \\
\hline $\begin{array}{l}\text { Chigateri et al } \\
{[75]}\end{array}$ & $\begin{array}{l}\text { Frail elderly people } \\
\text { from independent- } \\
\text { living retirement } \\
\text { homes }\end{array}$ & $23(6 / 17)$ & $\begin{array}{l}\text { WIS } 1 \text { triaxial ac- } \\
\text { celerometer (L5 verte- } \\
\text { bra) }\end{array}$ & $\begin{array}{l}\text { TUGT, } \mathrm{STS}^{\mathrm{j}} \text {, activities of daily liv- } \\
\text { ing }\end{array}$ & $\begin{array}{l}\text { Synchronized videos } \\
\text { with accelerometer (identifica- } \\
\text { tion of the beginning of } \\
\text { TUGT and of walking } \\
\text { episode) }\end{array}$ \\
\hline Qiu et al [76] & $\begin{array}{l}\mathrm{CDEP} / \text { social wel- } \\
\text { fare centers }\end{array}$ & $196(0 / 196)$ & $\begin{array}{l}\text { WIS } 5 \text { sensors with 3- } \\
\text { axis-acceleration, 3- } \\
\text { axis angular velocity, } \\
\text { 3-axis magnetism } \\
\text { each (low back, upper } \\
\text { legs, lower legs) }\end{array}$ & $\begin{array}{l}\text { Sensory integration test, limits of } \\
\text { stability forward reach, STS-5, } \\
\text { TUGT, motor function }\end{array}$ & $\begin{array}{l}\text { Classification of faller based } \\
\text { on self-reported history }\end{array}$ \\
\hline Hiesh et al [77] & $\begin{array}{l}\text { Healthy elderly } \\
\text { people }\end{array}$ & $30(12 / 18)$ & $\begin{array}{l}\text { Smartphone technolo- } \\
\text { gy, } 1 \text { accelerometer } \\
\text { (sternum) }\end{array}$ & $\begin{array}{l}\text { Balance tests standing on a force } \\
\text { plate and holding a smartphone } \\
\text { against the chest: eyes open/closed } \\
\text { DT, semitandem, tandem stance, } \\
\text { single-leg stance }\end{array}$ & $\begin{array}{l}\text { Comparison between force } \\
\text { plate and smartphone data }\end{array}$ \\
\hline $\begin{array}{l}\text { Nightingale et } \\
\text { al [79] }\end{array}$ & $\begin{array}{l}\text { Local community } \\
\text { centers/health care } \\
\text { provider offices/se- } \\
\text { nior centers }\end{array}$ & 51 (unknown) & $\begin{array}{l}\text { OptoGait system pho- } \\
\text { toelectric technology }\end{array}$ & $10 \mathrm{~m}$ walk & TUGT \\
\hline
\end{tabular}

${ }^{\mathrm{a}} \mathrm{WIS}$ : wearable inertial sensor.

${ }^{\mathrm{b}}$ TUGT: Timed Up and Go Test.

${ }^{\mathrm{c} C D E P}$ : community-dwelling elderly people. 
${ }^{\mathrm{d}}$ ST: single task.

${ }^{\mathrm{e}}$ DT: dual task.

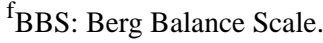

gFES: Fall Efficacy Scale.

${ }^{\mathrm{h}}$ STS-5: Five Times Sit-to-Stand test.

${ }^{\mathrm{i}}$ AST: Alternative Step Test.

${ }^{j}$ STS: Sit-to-Stand test.

\section{Focus of eHealth Services}

The 12 QESs were focused on screening and assessment. These studies compared the use of sensors in participants with a history of falling, or with clinical tests and functional scales
[66,68-77,79] (Table 5). No instrumental test of the inner ear was performed as a gold-standard test. All RCTs [59-65] and two QESs [67,78] were focused on balance treatment or rehabilitation (Table 6). Again, no instrumental test of the inner ear was used in pre and postrehabilitation assessments. 
Table 6. Studies focused on treatment/rehabilitation.

\begin{tabular}{|c|c|c|c|c|c|c|c|}
\hline Reference & $\begin{array}{l}\text { Study } \\
\text { type }\end{array}$ & $\begin{array}{l}\text { Setting and } \\
\text { population }\end{array}$ & $\begin{array}{l}\text { Participants, } \mathrm{N} \\
\text { (male/female) }\end{array}$ & Tested technology & $\begin{array}{l}\text { Tested sensor archi- } \\
\text { tecture }\end{array}$ & $\begin{array}{l}\text { Sensor-based proce- } \\
\text { dures }\end{array}$ & $\begin{array}{l}\text { Outcome mea- } \\
\text { surements }\end{array}$ \\
\hline $\begin{array}{l}\text { Eggenberger } \\
\text { et al [59] }\end{array}$ & $\mathrm{RCT}^{\mathrm{a}}$ & $\begin{array}{l}\mathrm{CDEP}^{\mathrm{b}} \text { and } \\
\text { retirement } \\
\text { homes }\end{array}$ & $\begin{array}{l}71(25 / 46): \text { dance } \\
\text { group } n=24, \text { memory } \\
\text { group } n=22, \text { control } \\
\text { group } n=25\end{array}$ & $\begin{array}{l}\mathrm{VR}^{\mathrm{c}} \text { video game } \\
\text { dancing + Impact } \\
\text { Dance Platform } \\
\text { treadmill walking + } \\
\text { computer screen + } \\
\text { training software }\end{array}$ & $\begin{array}{l}\text { VR video game } \\
\text { dancing + pressure } \\
\text { sensitive platform, } \\
\text { treadmill + training } \\
\text { software }\end{array}$ & $\begin{array}{l}\text { VR video game } \\
\text { dancing with simulta- } \\
\text { neous cognitive- } \\
\text { physical training; } \\
\text { treadmill walking } \\
\text { with simultaneous } \\
\text { verbal memory } \\
\text { training; treadmill } \\
\text { walking (control) }\end{array}$ & $\begin{array}{l}\text { Gait analysis: } \\
\mathrm{ST}^{\mathrm{d}} / \mathrm{DT}^{\mathrm{e}} 7.3 \mathrm{~m} \\
\text { walking, Short } \\
\text { Physical Perfor- } \\
\text { mance Battery, } \\
\text { fall frequency, 6- } \\
\text { minute walk test, } \\
\text { measure of fall } \\
\text { fear }\end{array}$ \\
\hline $\begin{array}{l}\text { Gschwind et } \\
\text { al [60] }\end{array}$ & RCT & CDEP & $\begin{array}{l}153(60 / 93) \text { : interven- } \\
\text { tion group } n=78, \text { con- } \\
\text { trol group } n=75\end{array}$ & $\begin{array}{l}\text { iStoppFalls system: } \\
\text { computer + Google } \\
\text { TV set top box + } \\
\text { Microsoft Kinect + } \\
\text { senior mobility } \\
\text { monitor + android } \\
\text { tablet }\end{array}$ & $\begin{array}{l}\text { Kinect-based sys- } \\
\text { tem (3D depth sen- } \\
\text { sor), Senior Mobil- } \\
\text { ity Monitor (3D } \\
\text { accelerometer, } \\
\text { barometer) }\end{array}$ & $\begin{array}{l}\text { 16-week home- } \\
\text { based balance ex- } \\
\text { ergames and muscle } \\
\text { strength exercises + } \\
\text { education booklet } \\
\text { (intervention) or edu- } \\
\text { cation booklet + } \\
\text { usual activities (con- } \\
\text { trol) }\end{array}$ & $\begin{array}{l}\text { Estimated risk of } \\
\text { falling; mobility, } \\
\text { self-care, usual } \\
\text { activities, pain, } \\
\text { discomfort, anxi- } \\
\text { ety, depression, } \\
\text { health question- } \\
\text { naires, cognitive } \\
\text { performance, } \\
\text { walking task, } \\
\text { STS-5 }{ }^{\mathrm{f}}, \text { TUGT } \\
\text { technology use }\end{array}$ \\
\hline $\begin{array}{l}\text { Gschwind et } \\
\text { al [61] }\end{array}$ & RCT & CDEP & $\begin{array}{l}124(42 / 82) \text { : step-mat } \\
\text { training group } n=39, \\
\text { Microsoft-Kinect } \\
\text { group } n=24, \text { control } \\
\text { group } n=61\end{array}$ & $\begin{array}{l}\text { Input device, com- } \\
\text { puter, USB modem, } \\
\text { TV, exergames, Mi- } \\
\text { crosoft Kinect or } \\
\text { electronic mat }\end{array}$ & $\begin{array}{l}\text { Pressure-sensitive } \\
\text { electronic mat, } \\
\text { Kinect-based sys- } \\
\text { tem (3D depth sen- } \\
\text { sor) }\end{array}$ & $\begin{array}{l}\text { Unsupervised 16- } \\
\text { week home exercise } \\
\text { using exergames or } \\
\text { educational booklet } \\
\text { about evidence- } \\
\text { based health and fall } \\
\text { prevention advice + } \\
\text { usual activities (con- } \\
\text { trol) }\end{array}$ & $\begin{array}{l}\text { Risk of falling, } \\
\text { health and disabil- } \\
\text { ity measure, STS- } \\
5, \text { TUGT, cogni- } \\
\text { tive performance }\end{array}$ \\
\hline $\begin{array}{l}\text { Hall et al } \\
{[67]}\end{array}$ & $\mathrm{QES}^{\mathrm{h}}$ & CDEP & $\begin{array}{l}16(0 / 16): \text { group A } \\
n=8, \text { group } B n=8\end{array}$ & $\begin{array}{l}\text { Nintendo Wii Fit } \\
\text { System: computer } \\
\text { interface + monitor } \\
\text { + Wii balance board } \\
\text { + games Ski Slalom/ } \\
\text { Table Tilt }\end{array}$ & $\begin{array}{l}\text { Balance Board: } \\
\text { force platform }\end{array}$ & $\begin{array}{l}\text { Wii Fit balance test } \\
+ \text { games, followed } \\
\text { by SOT }{ }^{\mathrm{i}} \text { and LOS } \\
\text { test, CDP } \text { (group }^{\mathrm{j}} \\
\text { A); SOT and LOS } \\
\text { test, CDP, followed } \\
\text { by Wii Fit balance } \\
\text { test + games (group } \\
\text { B) }\end{array}$ & $\begin{array}{l}\text { Dynamic Gait In- } \\
\text { dex, TUGT, gait } \\
\text { speed }\end{array}$ \\
\hline $\begin{array}{l}\text { Lim et al } \\
{[62]}\end{array}$ & $\mathrm{RCT}$ & CDEP & $\begin{array}{l}36(11 / 25) \text { : interven- } \\
\text { tion group } n=18, \text { con- } \\
\text { trol group } n=18 \text {. }\end{array}$ & $\begin{array}{l}\text { Wearable balance } \\
\text { biofeedback (system } \\
\text { (vibrotactile, audito- } \\
\text { ry and visual } \\
\text { biofeedback) }\end{array}$ & $\begin{array}{l}\text { Biofeedback head- } \\
\text { band: } 8 \text { vibrotactile } \\
\text { actuators, } 2 \text { bone- } \\
\text { conducting acous- } \\
\text { tic transducers, } 3 \\
\text { light-emitting } \\
\text { diodes; and gyro- } \\
\text { scopes (lower } \\
\text { back) }\end{array}$ & $\begin{array}{l}\text { 2-week training with } \\
\text { real-time multi- } \\
\text { modal biofeedback } \\
\text { of trunk sway or 2- } \\
\text { week training with- } \\
\text { out biofeedback } \\
\text { (control) }\end{array}$ & $\begin{array}{l}\text { Standing: } 1 \text { leg } \\
\text { (eyes open), feet } \\
\text { together, firm } \\
\text { surface and foam, } \\
\text { tandem stance } \\
\left(\mathrm{EC}^{\mathrm{l}}\right) \text {; self-paced } \\
8 \mathrm{~m} \text { walking } \\
(\mathrm{EC}) ; 8 \mathrm{~m} \text { walk- } \\
\text { ing with head } \\
\text { turning; } 8 \text { tandem } \\
\text { steps (EC) }\end{array}$ \\
\hline $\begin{array}{l}\text { Oesch et al } \\
{[63]}\end{array}$ & $\mathrm{RCT}$ & $\begin{array}{l}\text { Geriatric reha- } \\
\text { bilitation cen- } \\
\text { ter }\end{array}$ & $\begin{array}{l}54(29 / 25) \text { : interven- } \\
\text { tion group } n=26, \text { con- } \\
\text { trol group } n=28\end{array}$ & $\begin{array}{l}\text { Windows Kinect } \\
\text { (exergame) }\end{array}$ & $\begin{array}{l}\text { Kinect-based sys- } \\
\text { tem ( } 3 D \text { depth sen- } \\
\text { sor) }\end{array}$ & $\begin{array}{l}\text { 10-day self-regulat- } \\
\text { ed training with ex- } \\
\text { ergames or 10-day } \\
\text { self-regulated con- } \\
\text { ventional training } \\
\text { with instruction } \\
\text { leaflets (control) }\end{array}$ & $\begin{array}{l}\text { Adherence, moti- } \\
\text { vation, enjoy- } \\
\text { ment, sensor- } \\
\text { based walking } \\
\text { test }\end{array}$ \\
\hline
\end{tabular}




\begin{tabular}{|c|c|c|c|c|c|c|c|}
\hline Reference & $\begin{array}{l}\text { Study } \\
\text { type }\end{array}$ & $\begin{array}{l}\text { Setting and } \\
\text { population }\end{array}$ & $\begin{array}{l}\text { Participants, } \mathrm{N} \\
\text { (male/female) }\end{array}$ & Tested technology & $\begin{array}{l}\text { Tested sensor archi- } \\
\text { tecture }\end{array}$ & $\begin{array}{l}\text { Sensor-based proce- } \\
\text { dures }\end{array}$ & $\begin{array}{l}\text { Outcome mea- } \\
\text { surements }\end{array}$ \\
\hline $\begin{array}{l}\text { Ozaki et al } \\
{[64]}\end{array}$ & RCT & $\begin{array}{l}\text { Prefrail or } \\
\text { frail CDEP }\end{array}$ & $\begin{array}{l}27(7 / 20) \text { : intervention } \\
\text { group } n=14, \text { control } \\
\text { group } n=13\end{array}$ & $\begin{array}{l}\text { BEAR }^{\mathrm{m}} \text { system: } \\
\text { Stand-and-ride } \\
\text { transport robot + } \\
\text { wearable helmet and } \\
\text { suspending device + } \\
\text { software }\end{array}$ & $\begin{array}{l}\text { Stand-and-ride } \\
\text { transport robot } \\
\text { with two inverted } \\
\text { wheel motors }\end{array}$ & $\begin{array}{l}\text { 6-week based BEAR } \\
\text { training first group } \\
\text { or 6-week based } \\
\text { conventional bal- } \\
\text { ance training first } \\
\text { group (control) }\end{array}$ & $\begin{array}{l}\text { Gait speed, tan- } \\
\text { dem gait speed, } \\
\text { functional reach } \\
\text { test, TUGT }\end{array}$ \\
\hline $\begin{array}{l}\text { Hong et al } \\
{[65]}\end{array}$ & RCT & CDEP & $\begin{array}{l}23(0 / 23) \text { : intervention } \\
\text { group } n=10, \text { control } \\
\text { group } n=13\end{array}$ & $\begin{array}{l}\text { tablet, web app, sig- } \\
\text { naling server module } \\
\text { network address } \\
\text { translator traversal } \\
\text { module }\end{array}$ & $\begin{array}{l}\text { Web Real-Time } \\
\text { Communication } \\
\text { (WebRTC) technol- } \\
\text { ogy }\end{array}$ & $\begin{array}{l}\text { 12-week telepres- } \\
\text { ence exercise ses- } \\
\text { sions or maintained } \\
\text { lifestyle (control) }\end{array}$ & $\begin{array}{l}\text { Senior fitness } \\
\text { test, } \mathrm{BBS}^{\mathrm{n}} \text {, fall- } \\
\text { related self-effica- } \\
\text { cy, } \mathrm{FES}^{\mathrm{o}}, \text { Fear of } \\
\text { falling question- } \\
\text { naire }\end{array}$ \\
\hline
\end{tabular}

${ }^{\mathrm{a}} \mathrm{RCT}$ : randomized controlled trial.

${ }^{\mathrm{b}} \mathrm{CDEP}$ : community-dwelling elderly people.

${ }^{\mathrm{c}}$ VR: virtual reality.

${ }^{\mathrm{d}} \mathrm{ST}$ : single task.

e DT: dual task.

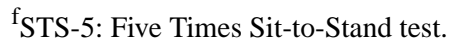

$\mathrm{g}_{\text {TUGT: Timed Up and Go Test }}$

${ }^{\mathrm{h}} \mathrm{QES}$ : quasiexperimental study.

${ }^{\mathrm{i}}$ SOT: Sensory Organization Test.

${ }^{\mathrm{j}}$ LOS: Limits of Stability.

${ }^{\mathrm{k}} \mathrm{CDP}$ : computerized dynamic posturography.

${ }^{1} \mathrm{EC}$ : eyes closed.

${ }^{m}$ BEAR: Balance Exercise Assist Robot.

${ }^{\mathrm{n}}$ BBS: Berg Balance Scale

${ }^{\circ}$ FES: Fall Efficacy Scale.

\section{Population Characteristics}

The included studies had large differences in sample sizes, ranging from 23 to 153 participants [59-65] among RCTs and from 16 to 196 participants [66-79] among QESs. As shown in Tables 5 and 6, many studies included a small sample size that was described as a limitation.

The age range was 60-91 years for the RCTs and 60-92 years for the QESs. Most of the studies included more women than men. In four studies, only women participated $[65,67,71,76]$. The decision to only recruit women was explained in one study as "to avoid the influence of gender differences on risk of falling" [76]. Two studies excluded the few male participants $[67,71]$ and the remaining article did not describe the reason for the exclusive participation of women [65]. One study did not describe the age range or the gender distribution of the participants [79].

The participants ( $\geq 60$ years old) were recruited from the community [60-62,64,65,67-70,72-74,76,77], gerontology services $[71,75,78]$, both $[59,79]$, or at a hospital [66]. One study included participants who were referred for geriatric inpatient rehabilitation [63].

Most of the studies did not describe the characteristics of health conditions of the sample [59,62,64-66,68-72,74-77,79]. Only some authors provided quantitative data about the participants' medication use $[60,61,73,78]$ and their comorbidities $[60,61,63,67,73,78]$. One study [60] highlighted the following comorbidities of the participants: heart problems, high blood pressure, osteoporosis, lower back pain, hip pain, knee and/or leg pain, and foot pain. Two studies excluded participants with self-reported balance disorders [62,63]. Two other studies included frail or prefrail elderly adults $[64,75]$.

\section{Balance Disorder and Identification of Faller Participants}

The included studies used functional balance tests, with or without sensors, to evaluate balance and risk of falling. An objective identification via exploration and quantification of the function of the inner ear by instrumental tests was not employed in any of the considered studies, as mentioned above. 
Therefore, the presence of prebyvestibulopathy or other balance disorders was not known.

Some authors highlighted the potential of sensor-based tests in identifying early balance deficits [71] and in evaluating the risk of falling [66,72,73,76,77]. Improved balance and gait with technology-based training were mentioned in some studies $[59,62,64]$

The identification of faller participants based on retrospective $[68,70,73,76]$ or prospective occurrence of falling [74] was employed to compare the technology results. The benefits of virtual training in reducing the risk of falling was also described $[60,61,65,78]$.

No study focusing on detection of falling fully complied with the inclusion criteria of this review (RCTs or QESs, published in English between January 2015 and December 2019, restricted to the population 60 years or older).

\section{eHealth Platform and Services}

Different platforms were used for the provision of eHealth services. The main platforms identified were computer-based apps, either via the internet or mobile based platforms (Tables 5 and 6).

As mentioned above, 12 studies focused on screening or assessment [66,68-77,79] using different types (wearable inertial, wearable pressure, pendant, smartphone), quantities (range 1-5), and locations (head, sternum, lumbar vertebra, pelvis, hip, leg, shanks, foot) of sensors. Single or combined sensor-based tasks were employed. Only two studies [73,75] evaluated activities in a real-life environment (Table 5).
Nine studies explored balance rehabilitation [59-65,67,78] with different exercises and duration of training. The follow-up time was short (less than 6 months) in most of the studies, with the longest follow-up of 1 year [59]. The development of eHealth services was explored both inside and outside the laboratory environment (Table 6).

One study used a robot to provide information about training and fall prevention. However, the authors pointed out that the screen and the volume speaker were not adequate for use by elderly people [78].

The use of technical language and the presence of disabilities such as visual and hearing impairment were highlighted as the main barriers in using eHealth [78].

\section{Health Benefits}

Only one study did not report better adherence, enjoyment, motivation, and balance performance with virtual training. This was explained by the possible fragility of the sample included in the study and by the short duration of the training intervention [63].

The remaining papers emphasized the potential contribution of digital solutions to improve balance performance and risk of falling. The sensors used during balance tests improved the evaluation of balance and gait $[66,68,69,71,72,79]$ and improved the identification of potential faller participants [70,73-77]. In addition, the use of eHealth devices for balance rehabilitation increased balance and gait performance [59,60,62,64,65,78], and reduced the risk of falling $[60,61]$. However, no long-term follow-up was reported. Virtual programs of falls prevention seemed to increase knowledge on the subject [78] (Table 7). 
Table 7. Health benefits: conclusions from all studies.

\begin{tabular}{|c|c|c|}
\hline Reference & Study type & Conclusions \\
\hline Zacaria et al [66] & $\mathrm{QES}^{\mathrm{a}}$, screening & Single wearable sensor during $\mathrm{TUGT}^{\mathrm{b}}$ : an improved tool in evaluating fall risk \\
\hline Howcroft et al [68] & QES, screening & Sensor-based gait assessment: potential of identification of gait changes \\
\hline Lee et al [69] & QES, screening & Advantages of wearable sensor as an outside laboratory tool \\
\hline Ponti et al [70] & QES, screening & $\begin{array}{l}\text { Improved potential of identification of fallers with single sensor-based } \mathrm{DT}^{\mathrm{c}} \\
\text { TUGT }\end{array}$ \\
\hline Similä et al [71] & QES, screening & Sensor-based walk test: a screening tool to identify early signs of balance deficits \\
\hline Shahzad et al [72] & QES, screening & Importance of sensor-based TUGT, STS- $5^{\mathrm{d}}$, and $\mathrm{AST}^{\mathrm{e}}$ on fall risk estimation \\
\hline Brodie et al [73] & QES, multicenter screening & Better sensor-based daily-life gait assessment to discriminate fallers \\
\hline Howcroft et al [74] & QES, screening & $\begin{array}{l}\text { Sensor: potential to discriminate differences between } \mathrm{ST}^{\mathrm{f}} \text { and } \mathrm{DT} \text { gait and be- } \\
\text { tween prospective fallers and nonfallers }\end{array}$ \\
\hline Chigateri et al [75] & QES, screening & $\begin{array}{l}\text { Wearable accelerometer: useful for nonsedentary activity recognition and gait } \\
\text { detection in frail older adults outside lab facilities }\end{array}$ \\
\hline Qiu et al [76] & QES, screening & $\begin{array}{l}\text { Potential use of wearable inertial sensor-based systems for elderly fall risk as- } \\
\text { sessment }\end{array}$ \\
\hline Hiesh et al [77] & QES, screening & $\begin{array}{l}\text { Validity of smartphone for evaluation of postural stability and fall risk stratifica- } \\
\text { tion in older adults }\end{array}$ \\
\hline Nightingale et al [79] & QES, screening & Using Optogait system: TUGT as a tool for screening balance deficits \\
\hline Eggenberger et al [59] & $\mathrm{RCT}^{\mathrm{g}}$, rehabilitation & $\begin{array}{l}\text { Virtual reality game dancing with simultaneous cognitive-physical training and } \\
\text { treadmill walking with simultaneous verbal memory training: potential to enhance } \\
\text { gait variables }\end{array}$ \\
\hline Gschwind et al [60] & $\mathrm{RCT}$, rehabilitation & iStoppFalls program reduced physiological fall risk and improved postural sway \\
\hline Gschwind et al [61] & RCT, rehabilitation & $\begin{array}{l}\text { Step-mat-training and Microsoft-Kinect exergames reduced fall risks, Step-mat- } \\
\text { training improved specific cognitive functions; neither intervention improved } \\
\text { balance control }\end{array}$ \\
\hline Hall et al [67] & QES, rehabilitation & $\begin{array}{l}\text { WiiFit feasible to safely use, Ski Slalom game similar effect as computerized } \\
\text { dynamic posturography }\end{array}$ \\
\hline Lim et al [62] & RCT, rehabilitation & $\begin{array}{l}\text { Balance training with biofeedback: most beneficial for the most difficult tasks } \\
\text { but with few long-term benefits }\end{array}$ \\
\hline Oesch et al [63] & RCT, rehabilitation & $\begin{array}{l}\text { Superior results of conventional training with respect to adherence, enjoyment, } \\
\text { and motivation; no difference of balance during walking between conventional } \\
\text { and training with exergames }\end{array}$ \\
\hline Ozaki et al [64] & $\begin{array}{l}\text { Crossover trial without a } \\
\text { washout term, rehabilitation }\end{array}$ & $\begin{array}{l}\mathrm{BEAR}^{\mathrm{h}} \text { training more effective for improving dynamic balance and lower ex- } \\
\text { tremity muscle strength }\end{array}$ \\
\hline Hong et al [65] & RCT, rehabilitation & $\begin{array}{l}\text { Telepresence exercise program: effective to improve balance and reduce fear of } \\
\text { fall; no significant difference of fall efficacy between intervention (telepresence } \\
\text { exercise sessions) and control group (maintained lifestyle) }\end{array}$ \\
\hline Maneproom et al [78] & QES, rehabilitation & $\begin{array}{l}\text { Robotic fall prevention program increased fall prevention knowledge, promoted } \\
\text { exercises, and improved balance }\end{array}$ \\
\hline
\end{tabular}

${ }^{\mathrm{a}} \mathrm{QES}$ : quasiexperimental study.

b TUGT: Timed Up and Go Test.

${ }^{\mathrm{c}} \mathrm{DT}$ : dual task.

${ }^{\mathrm{d}}$ STS-5: Five Times Sit-to-Stand test.

eAST: Alternative Step Test.

${ }^{\mathrm{f}} \mathrm{ST}$ : single task.

${ }^{\mathrm{g}} \mathrm{RCT}$ : randomized controlled trial.

${ }^{\mathrm{h}}$ BEAR: Balance Exercise Assist Robot. 


\section{Fall Prevention Literacy}

None of the studies explored the previous health literacy of the participants. Only two papers described the educational level of the participants $[60,78]$.

One study compared use of a fall prevention software to a conventional handbook to evaluate the improvement of knowledge on fall prevention. Both the intervention and control groups showed improvement in knowledge, without a significant difference [78].

\section{Discussion}

\section{Principal Findings}

Population aging, and the associated vulnerability to the development of multiple chronic pathologies and balance disorders, have motivated research and the implementation of new strategies for the provision of health care. eHealth devices have been studied to help assess balance and gait performance, risk of falling in and outside a laboratory setting, and to perform in-home balance rehabilitation. In this review, we confirmed the potential of eHealth to complement the health care of elderly people. However, most of these studies were not designed to provide clinical guidelines.

Despite growing interest about this subject in the last 20 years, we decided to focus on studies published in the last 5 years (RCTs and QESs), taking into consideration both continuous advances in technological innovation and the opportunity to apply new clinical applications in balance disorder and risk of falling for the elderly population.

Unlike other reviews, our eligibility criteria ruled out many initially retrieved articles, especially studies with participants under 60 years old, those without reporting the age of participants, or with participants having a functional limitation due to neurologic disease. Therefore, only 21 articles fully complied with the requirements of this review [59-79].

Except for one study [63], the others showed the potential of eHealth to evaluate balance assessment and risk of falling of elderly people and to promote balance training. The eHealth devices allowed collecting additional information about the balance, gait, and risk of falling of elderly people, and to monitor their daily activities.

In particular, eHealth seems to provide an opportunity for increasing medical-patient interactions and to reduce access inequities [30]. In 1996, Viierre et al [80] had already mentioned the potential of eHealth in this field: "remote medical diagnosis and treatment facilities could make the few vestibular disorder specialists much more available to patients." However, as observed in other reviews [36,37,42-47], the differences in methodologies and of variables included in the studies did not allow for a proper comparison to guide clinical applicability.

First, there was a broad range of sample sizes, which were generally quite small (ranging from 16 to 196 participants). A small sample of participants is considered a limitation for extrapolating the results, especially for the exploration of risk of falling.
Second, there were missing data about the clinical conditions of the participants. Except for one study [60], several volunteers were recruited from the community and were defined as "healthy" elderly people only based on a self-reported assessment. There were also participants recruited from geriatric services without reference to their clinical conditions. Despite the exclusion of participants with self-reported balance disorders in two studies [62,63], we consider that the exclusion rules should be more rigorous and based on objective data such as instrumental inner ear tests. We have to take into consideration that elderly people can have instability due to many conditions, including the normative aging process, and therefore the outcomes from a balance rehabilitation intervention could be sensitive to these differences [38-41].

Third, different research methodologies were used for screening and assessment. We observed a wide range of clinical tests, functional scales, faller classifications, and sensor-based tasks among the included studies. The lack of homogeneity of these variables limited an appropriate comparison among the studies. Moreover, functional inner ear tests were not used as the gold-standard test. We consider this as a weakness common to all studies.

Fourth, different types of sensors were used for screening and assessment. Similar to the findings of other reviews [36,37,44-47], the studies employed mainly accelerometers, with variations in both number and body location.

Fifth, as observed previously [42,43], studies focusing on treatment and rehabilitation used different devices, training durations, and follow-up times. Some authors employed supervised training. In one study, this was used a telepresence-based exercise platform [65]. Others employed in-home self-regulated exercises training [61], thereby avoiding the need for participants to travel to the rehabilitation center. None of the studies described pre and postintervention data about the function of the inner ear. The studies did not verify the long-term effect of training, especially with respect to fall occurrence. Only two studies explored a sensor used in real-life activities [73,75], which is relevant since it allowed for a better evaluation of the remote interaction and monitoring of daily activities.

Additionally, we observed a constraint related to the use of devices that are not fully adequate to match the abilities of elderly people [78]. We also highlight the importance of providing a better definition of the eHealth user profile to improve adherence.

Future studies in this field should consider the above topics as a starting point, as well as for health policy implementations on eHealth apps for elderly people with balance disorders.

The use of eHealth can play an important role as a complementary method to provide health care services, encouraging health promotion and patient participation, as well as allowing for the remote management of balance disorders. 


\section{Recommendations}

Based on this review, we can provide the following recommendations to improve studies and applications of eHealth for preventing fall risk in the elderly population.

First, this review highlights the need for further research on the use of eHealth devices in proper clinical settings. This represents an opportunity to be explored, reaching out to elderly people with balance and risk of falling.

Second, despite several efforts to explore balance among the elderly, there is still a need for better characterization and description of the health condition of the population under study. In particular, we recommend future studies to include the results of functional tests of the inner ear as a gold-standard test or for comparison of the outcome before and after remote balance rehabilitation. Most of the interventions were developed with only functional balance tests. Future studies should also focus on the real-life environment, allowing for additional information of the daily activities among elderly participants.
Third, a longer follow-up time is important to evaluate the long-term benefits of eHealth tools on the balance performance and risk of falling of elderly people.

Finally, the eHealth devices should be user-friendly to improve adherence among elderly people.

\section{Limitations}

This review was limited to articles written in the English language and available on the PubMed, Google Scholar, Embase, and SciELO databases for the last 5 years; therefore, it is possible that relevant studies were missed.

\section{Conclusions}

The inclusion of eHealth services can play a critical role for the better provision of health care to elderly people with a balance disorder and risk of falling. The differences in populations, methodologies, eHealth devices, and follow-up times of the included studies did not allow for a clear comparison between results, therefore limiting the possibility of obtaining valid guidance for clinical applicability. More rigorous studies are recommended.

\section{Acknowledgments}

This work was partially supported by Fundação para a Ciência e a Tecnologia (FCT) for funds to Global Health and Tropical Medicine (GHTM) (UID/04413/2020 to LVL).

\section{Conflicts of Interest}

None declared.

\section{References}

1. Action plan on specific action on innovation in support of personalized health management, starting with a falls prevention initiative. European Innovation Partnership on Active and Healthy Ageing (EIP-AHA). 2013. URL: https://ec.europa.eu/ eip/ageing/library/action-plan-specific-action-innovation-support-personalized-health-management-starting-falls en.html [accessed 2020-01-05]

2. Global strategy and action plan on ageing and health. World Health Organization. 2017. URL: https://www.who.int/ageing/ WHO-GSAP-2017.pdf?ua=1 [accessed 2020-01-05]

3. Health at a glance 2019 - OECD INDICATORS. Organization for Economic Co-operation and Development (OECD). URL: https://oecd-ilibrary.org/social-issues-migration-health/health-at-a-glance-2019 4dd50c09-en [accessed 2020-05-17]

4. Ageing Europe - Looking at the lives of older people in the EU. Eurostat - Statistics Explained. 2020. URL: https://ec. europa.eu/eurostat/statistics-explained/index.php/Ageing_Europe - looking at the lives of older_people in the EU [accessed 2020-11-29]

5. Salzman B. Gait and balance disorders in older adults. Am Fam Physician 2010 Jul 01;82(1):61-68 [FREE Full text] [Medline: 20590073]

6. Agrawal Y, Van de Berg R, Wuyts F, Walther L, Magnusson M, Oh E, et al. Presbyvestibulopathy: Diagnostic Criteria Consensus document of the classification committee of the Bárány Society. J Vestib Res 2019;29(4):161-170. [doi: 10.3233/VES-190672] [Medline: 31306146]

7. WHO global report on falls prevention in older age. World Health Organization. 2007. URL: https://www.who.int/ageing/ publications/Falls prevention7March.pdf [accessed 2020-01-05]

8. Berry SD, Miller RR. Falls: epidemiology, pathophysiology, and relationship to fracture. Curr Osteoporos Rep 2008 Dec;6(4):149-154 [FREE Full text] [doi: 10.1007/s11914-008-0026-4] [Medline: 19032925]

9. Haagsma JA, Olij BF, Majdan M, van Beeck EF, Vos T, Castle CD, et al. Falls in older aged adults in 22 European countries: incidence, mortality and burden of disease from 1990 to 2017. Inj Prev 2020 Oct;26(Supp 1):i67-i74. [doi:

10.1136/injuryprev-2019-043347] [Medline: 32111726]

10. Integrated care for older people Guidelines on community-level interventions to manage declines in intrinsic capacity. World Health Organization Institutional Repository for Information Sharing. 2017. URL: https://apps.who.int/iris/handle/ $\underline{10665 / 258981}$ [accessed 2020-01-05] 
11. Neuhauser H. The epidemiology of dizziness and vertigo. Handb Clin Neurol 2016;137:67-82. [doi: 10.1016/B978-0-444-63437-5.00005-4] [Medline: 27638063]

12. Wojtczak R, Narożny W, Kuczkowski J, Siebert J. Epidemiology of dizziness in northern Poland - The first Polish neurootologic survey of the general population. Ann Agric Environ Med 2017 Sep 21;24(3):502-506. [doi: 10.5604/12321966.1228401] [Medline: 28954498]

13. Fernández L, Breinbauer HA, Delano PH. Vertigo and dizziness in the elderly. Front Neurol 2015;6:144. [doi: 10.3389/fneur.2015.00144] [Medline: 26167157]

14. Kerber KA, Callaghan BC, Telian SA, Meurer WJ, Skolarus LE, Carender W, et al. Dizziness symptom type prevalence and overlap: A US nationally representative survey. Am J Med 2017 Dec;130(12):1465.e1-1465.e9. [doi: 10.1016/j.amjmed.2017.05.048] [Medline: 28739195]

15. Lin HW, Bhattacharyya N. Balance disorders in the elderly: epidemiology and functional impact. Laryngoscope 2012 Aug;122(8):1858-1861. [doi: 10.1002/lary.23376] [Medline: 22645067]

16. Maarsingh OR, Dros J, Schellevis FG, van Weert HC, Bindels PJ, Horst HEVD. Dizziness reported by elderly patients in family practice: prevalence, incidence, and clinical characteristics. BMC Fam Pract 2010 Jan 11;11:2 [FREE Full text] [doi: 10.1186/1471-2296-11-2] [Medline: 20064231]

17. Gillespie LD, Robertson MC, Gillespie WJ, Sherrington C, Gates S, Clemson LM, et al. Interventions for preventing falls in older people living in the community. Cochrane Database Syst Rev 2012 Sep 12(9):CD007146. [doi:

10.1002/14651858.CD007146.pub3] [Medline: 22972103]

18. Ekvall Hansson E, Magnusson M. Vestibular asymmetry predicts falls among elderly patients with multi-sensory dizziness. BMC Geriatr 2013 Jul 22;13:77 [FREE Full text] [doi: 10.1186/1471-2318-13-77] [Medline: 23875891]

19. Kristinsdottir EK, Jarnlo GB, Magnusson M. Asymmetric vestibular function in the elderly might be a significant contributor to hip fractures. Scand J Rehabil Med 2000 Jun;32(2):56-60. [Medline: 10853718]

20. Kristinsdottir EK, Nordell E, Jarnlo GB, Tjäder A, Thorngren KG, Magnusson M. Observation of vestibular asymmetry in a majority of patients over 50 years with fall-related wrist fractures. Acta Otolaryngol 2001 Jun;121(4):481-485. [Medline: 11508508]

21. Baldursdottir B, Petersen H, Jonsson PV, Mogensen B, Whitney SL, Ramel A, et al. Sensory impairments and wrist fractures: A case-control study. J Rehabil Med 2018 Feb 13;50(2):209-215 [FREE Full text] [doi: 10.2340/16501977-2312] [Medline: 29271981]

22. Heinrich S, Rapp K, Rissmann U, Becker C, König HH. Cost of falls in old age: a systematic review. Osteoporos Int 2010 Jun;21(6):891-902. [doi: 10.1007/s00198-009-1100-1] [Medline: 19924496]

23. Kerber KA. Vertigo and dizziness in the emergency department. Emerg Med Clin North Am 2009 Feb;27(1):39-50 [FREE Full text] [doi: $\underline{10.1016 / j . e m c .2008 .09 .002]}$ [Medline: $\underline{19218018}$ ]

24. Kovacs E, Wang X, Grill E. Economic burden of vertigo: a systematic review. Health Econ Rev 2019 Dec 27;9(1):37 [FREE Full text] [doi: 10.1186/s13561-019-0258-2] [Medline: 31883042]

25. Saber Tehrani AS, Coughlan D, Hsieh YH, Mantokoudis G, Korley FK, Kerber KA, et al. Rising annual costs of dizziness presentations to U.S. emergency departments. Acad Emerg Med 2013 Jul;20(7):689-696. [doi: 10.1111/acem.12168] [Medline: 23859582]

26. Bhaskar S, Bradley S, Chattu VK, Adisesh A, Nurtazina A, Kyrykbayeva S, et al. Telemedicine across the Globe-Position Paper from the COVID-19 Pandemic Health System Resilience PROGRAM (REPROGRAM) International Consortium (Part 1). Front Public Health 2020;8:556720. [doi: 10.3389/fpubh.2020.556720] [Medline: 33178656]

27. Gammon D, Berntsen GKR, Koricho AT, Sygna K, Ruland C. The chronic care model and technological research and innovation: a scoping review at the crossroads. J Med Internet Res 2015 Feb 06;17(2):e25 [FREE Full text] [doi: 10.2196/jmir.3547] [Medline: 25677200]

28. Kim K, Gollamudi SS, Steinhubl S. Digital technology to enable aging in place. Exp Gerontol 2017 Feb;88:25-31. [doi: 10.1016/j.exger.2016.11.013] [Medline: 28025126]

29. Global Observatory of eHealth. Global diffusion of eHealth: Making universal health coverage achievable: Report of the third Global Observatory on eHealth. World Health Organization. 2016. URL: https://apps.who.int/iris/bitstream/handle/ 10665/252529/9789241511780-eng.pdf;jsessionid=4857CD1FAD00D6536337F2868A07FD81? sequence=1\#page=16 [accessed 2019-11-07]

30. Lapão L, Dussault G. The contribution of eHealth and mHealth to improving the performance of the health workforce: a review. Public Health Panorama.: World Health Organization Regional Office for Europe; 2017. URL: https://apps.who.int/ iris/handle/10665/325262 [accessed 2019-11-07]

31. eHealth at WHO. World Health Organization. 2019 Dec 3. URL: https://www.who.int/ehealth/about/en/ [accessed 2019-12-03]

32. Gordon NP, Crouch E. Digital information technology use and patient preferences for internet-based health education modalities: cross-sectional survey study of middle-aged and older adults with chronic health conditions. JMIR Aging 2019 Apr 04;2(1):e12243 [FREE Full text] [doi: 10.2196/12243] [Medline: 31518291]

33. Merkel S, Hess M. The use of internet-based health and care services by elderly people in Europe and the importance of the country context: multilevel study. JMIR Aging 2020 Jun 03;3(1):e15491 [FREE Full text] [doi: 10.2196/15491] [Medline: 32490837] 
34. Ammenwerth E, Duftschmid G, Al-Hamdan Z, Bawadi H, Cheung NT, Cho K, et al. International comparison of six basic eHealth indicators across 14 countries: an eHealth benchmarking study. Methods Inf Med 2020 Dec;59(S 02):e46-e63 [FREE Full text] [doi: 10.1055/s-0040-1715796] [Medline: 33207386]

35. Yelnik A, Bonan I. Clinical tools for assessing balance disorders. Neurophysiol Clin 2008 Dec;38(6):439-445. [doi: 10.1016/j.neucli.2008.09.008] [Medline: 19026963]

36. Montesinos L, Castaldo R, Pecchia L. Wearable inertial sensors for fall risk assessment and prediction in older adults: a systematic review and meta-analysis. IEEE Trans Neural Syst Rehabil Eng 2018 Mar;26(3):573-582. [doi: 10.1109/TNSRE.2017.2771383] [Medline: 29522401]

37. Bet P, Castro PC, Ponti MA. Fall detection and fall risk assessment in older person using wearable sensors: A systematic review. Int J Med Inform 2019 Oct;130:103946. [doi: 10.1016/j.ijmedinf.2019.08.006] [Medline: 31450081]

38. Moreira Bittar RS, Bovino Pedalini ME, Oiticica Ramalho J, Yoshimura R. Critical analysis of vestibular rehabilitation outcome according to dizziness etiology. Braz J Otorhinolaryngol 2007 Nov;73(6):760-764. [doi: $10.1016 / \mathrm{s} 1808-8694(15) 31172-1]$

39. Han BI, Song HS, Kim JS. Vestibular rehabilitation therapy: review of indications, mechanisms, and key exercises. J Clin Neurol 2011 Dec;7(4):184-196 [FREE Full text] [doi: 10.3988/jen.2011.7.4.184] [Medline: 22259614]

40. Lacour M, Bernard-Demanze L. Interaction between vestibular compensation mechanisms and vestibular rehabilitation therapy: 10 recommendations for optimal functional recovery. Front Neurol 2014;5:285. [doi: 10.3389/fneur.2014.00285] [Medline: 25610424]

41. Rossi-Izquierdo M, Gayoso-Diz P, Santos-Pérez S, Del-Río-Valeiras M, Faraldo-García A, Vaamonde-Sánchez-Andrade I, et al. Vestibular rehabilitation in elderly patients with postural instability: reducing the number of falls-a randomized clinical trial. Aging Clin Exp Res 2018 Nov;30(11):1353-1361. [doi: 10.1007/s40520-018-1003-0] [Medline: 30008159]

42. Skjæret N, Nawaz A, Morat T, Schoene D, Helbostad JL, Vereijken B. Exercise and rehabilitation delivered through exergames in older adults: An integrative review of technologies, safety and efficacy. Int J Med Inform 2016 Jan;85(1):1-16. [doi: 10.1016/j.ijmedinf.2015.10.008] [Medline: 26559887]

43. Choi SD, Guo L, Kang D, Xiong S. Exergame technology and interactive interventions for elderly fall prevention: A systematic literature review. Appl Ergon 2017 Nov;65:570-581. [doi: 10.1016/j.apergo.2016.10.013] [Medline: 27825723]

44. Sun R, Sosnoff JJ. Novel sensing technology in fall risk assessment in older adults: a systematic review. BMC Geriatr 2018 Jan 16;18(1):14 [FREE Full text] [doi: 10.1186/s12877-018-0706-6] [Medline: 29338695]

45. Rucco R, Sorriso A, Liparoti M, Ferraioli G, Sorrentino P, Ambrosanio M, et al. Type and location of wearable sensors for monitoring falls during static and dynamic tasks in healthy elderly: a review. Sensors (Basel) 2018 May 18;18(5):1613 [FREE Full text] [doi: 10.3390/s18051613] [Medline: 29783647]

46. Nguyen H, Mirza F, Naeem MA, Baig MM. Falls management framework for supporting an independent lifestyle for older adults: a systematic review. Aging Clin Exp Res 2018 Nov;30(11):1275-1286. [doi: 10.1007/s40520-018-1026-6] [Medline: 30196346]

47. Leirós-Rodríguez R, García-Soidán JL, Romo-Pérez V. Analyzing the use of accelerometers as a method of early diagnosis of alterations in balance in elderly people: a systematic review. Sensors (Basel) 2019 Sep 09;19(18):3883 [FREE Full text] [doi: 10.3390/s19183883] [Medline: 31505828]

48. Liberati A, Altman DG, Tetzlaff J, Mulrow C, Gøtzsche PC, Ioannidis JPA, et al. The PRISMA statement for reporting systematic reviews and meta-analyses of studies that evaluate health care interventions: explanation and elaboration. PLoS Med 2009 Jul 21;6(7):e1000100 [FREE Full text] [doi: 10.1371/journal.pmed.1000100] [Medline: 19621070]

49. O'Connor D, Green S, Higgins JPT. Chapter 5: Defining the review questiondeveloping criteria for including studies. In: Higgins JPT, Green S, editors. Cochrane Handbook of Systematic Reviews of Intervention. Version 5.1.0 (updated March 2011). London, UK: The Cochrane Collaboration; 2011.

50. Active ageing: a policy framework. World Health Organization. 2002. URL: https://www.who.int/ageing/publications/ active ageing/en/ [accessed 2019-11-05]

51. Knudson D. Fundamentals of Biomechanics. 2nd edition. New York: Springer; 2007.

52. Rosso AL, Studenski SA, Chen WG, Aizenstein HJ, Alexander NB, Bennett DA, et al. Aging, the central nervous system, and mobility. J Gerontol A Biol Sci Med Sci 2013 Nov;68(11):1379-1386 [FREE Full text] [doi: 10.1093/gerona/glt089] [Medline: 23843270]

53. A health telematics policy in support of WHO's Health-for-all strategy for global health development: report of the WHO Group Consultation on Health Telematics, 11-16 December, Geneva, 1997. World Health Organization. 1998. URL: https:/ /apps.who.int/iris/handle/10665/63857 [accessed 2021-04-15]

54. Eysenbach G. What is e-health? J Med Internet Res 2001;3(2):E20 [FREE Full text] [doi: 10.2196/jmir.3.2.e20] [Medline: 11720962]

55. Deldar K, Bahaadinbeigy K, Tara SM. Teleconsultation and clinical decision making: a systematic review. Acta Inform Med 2016 Jul 16;24(4):286-292 [FREE Full text] [doi: 10.5455/aim.2016.24.286-292] [Medline: 27708494]

56. Jones DP. Biomedical Sensors. New York: Momentum Press; 2010.

57. Critical appraisal tools. The Joanna Briggs Institute. URL: https://jbi.global/critical-appraisal-tools [accessed 2019-01-05] 
58. Julian PT Higgins, Jelena Savovic, Matthew J Page, Jonathan AC Sterne on behalf of the ROB2 Development Group. Revised Cochrane risk-of-bias tool for randomized trials (Rob2). URL: https://sites.google.com/site/riskofbiastool/welcome/ rob-2-0-tool/current-version-of-rob-2 [accessed 2019-01-05]

59. Eggenberger P, Theill N, Holenstein S, Schumacher V, de Bruin ED. Multicomponent physical exercise with simultaneous cognitive training to enhance dual-task walking of older adults: a secondary analysis of a 6-month randomized controlled trial with 1-year follow-up. Clin Interv Aging 2015;10:1711-1732 [FREE Full text] [doi: 10.2147/CIA.S91997] [Medline: 26604719]

60. Gschwind YJ, Eichberg S, Ejupi A, de Rosario H, Kroll M, Marston HR, et al. ICT-based system to predict and prevent falls (iStoppFalls): results from an international multicenter randomized controlled trial. Eur Rev Aging Phys Act 2015;12:10 [FREE Full text] [doi: 10.1186/s11556-015-0155-6] [Medline: 26865874]

61. Gschwind YJ, Schoene D, Lord SR, Ejupi A, Valenzuela T, Aal K, et al. The effect of sensor-based exercise at home on functional performance associated with fall risk in older people - a comparison of two exergame interventions. Eur Rev Aging Phys Act 2015;12:11 [FREE Full text] [doi: 10.1186/s11556-015-0156-5] [Medline: 26865875]

62. Lim SB, Horslen BC, Davis JR, Allum JHJ, Carpenter MG. Benefits of multi-session balance and gait training with multi-modal biofeedback in healthy older adults. Gait Posture 2016 Jun;47:10-17. [doi: 10.1016/j.gaitpost.2016.03.017] [Medline: 27264396]

63. Oesch P, Kool J, Fernandez-Luque L, Brox E, Evertsen G, Civit A, et al. Exergames versus self-regulated exercises with instruction leaflets to improve adherence during geriatric rehabilitation: a randomized controlled trial. BMC Geriatr 2017 Mar 23;17(1):77 [FREE Full text] [doi: 10.1186/s12877-017-0467-7] [Medline: 28330455]

64. Ozaki K, Kondo I, Hirano S, Kagaya H, Saitoh E, Osawa A, et al. Training with a balance exercise assist robot is more effective than conventional training for frail older adults. Geriatr Gerontol Int 2017 Nov;17(11):1982-1990. [doi: 10.1111/ggi.13009] [Medline: 28295912]

65. Hong J, Kong H, Yoon H. Web-based telepresence exercise program for community-dwelling elderly women with a high risk of falling: randomized controlled trial. JMIR Mhealth Uhealth 2018 May 28;6(5):e132 [FREE Full text] [doi: 10.2196/mhealth.9563] [Medline: 29807877]

66. Zakaria NA, Kuwae Y, Tamura T, Minato K, Kanaya S. Quantitative analysis of fall risk using TUG test. Comput Methods Biomech Biomed Engin 2015;18(4):426-437. [doi: 10.1080/10255842.2013.805211] [Medline: 23964848]

67. Hall CD, Clevenger CK, Wolf RA, Lin JS, Johnson TM, Wolf SL. Feasibility of a low-cost, interactive gaming system to assess balance in older women. J Aging Phys Act 2016 Jan;24(1):111-118. [doi: 10.1123/japa.2014-0184] [Medline: $\underline{26181324]}$

68. Howcroft J, Kofman J, Lemaire ED, McIlroy WE. Analysis of dual-task elderly gait in fallers and non-fallers using wearable sensors. J Biomech 2016 May 03;49(7):992-1001. [doi: 10.1016/j.jbiomech.2016.01.015] [Medline: 26994786]

69. Lee C, Sun T, Jiang B, Choi V. Using wearable accelerometers in a community service context to categorize falling behavior. Entropy 2016 Jul 13;18(7):257 [FREE Full text] [doi: 10.3390/e18070257]

70. Ponti M, Bet P, Oliveira CL, Castro PC. Better than counting seconds: Identifying fallers among healthy elderly using fusion of accelerometer features and dual-task Timed Up and Go. PLoS One 2017;12(4):e0175559 [FREE Full text] [doi: 10.1371/journal.pone.0175559] [Medline: 28448509]

71. Similä H, Immonen M, Ermes M. Accelerometry-based assessment and detection of early signs of balance deficits. Comput Biol Med 2017 Jun 01;85:25-32. [doi: 10.1016/j.compbiomed.2017.04.009] [Medline: 28432935]

72. Shahzad A, Ko S, Lee S, Lee J, Kim K. Quantitative assessment of balance impairment for fall-risk estimation using wearable triaxial accelerometer. IEEE Sensors J 2017 Oct 15;17(20):6743-6751. [doi: 10.1109/jsen.2017.2749446]

73. Brodie MA, Coppens MJ, Ejupi A, Gschwind YJ, Annegarn J, Schoene D, et al. Comparison between clinical gait and daily-life gait assessments of fall risk in older people. Geriatr Gerontol Int 2017 Nov;17(11):2274-2282. [doi: 10.1111/ggi.12979] [Medline: 28176431]

74. Howcroft J, Lemaire ED, Kofman J, McIlroy WE. Dual-task elderly gait of prospective fallers and non-fallers: a wearable-sensor based analysis. Sensors (Basel) 2018 Apr 21;18(4):1275 [FREE Full text] [doi: 10.3390/s18041275] [Medline: 29690496]

75. Chigateri NG, Kerse N, Wheeler L, MacDonald B, Klenk J. Validation of an accelerometer for measurement of activity in frail older people. Gait Posture 2018 Oct;66:114-117. [doi: 10.1016/j.gaitpost.2018.08.024] [Medline: 30172217]

76. Qiu H, Rehman RZU, Yu X, Xiong S. Application of wearable inertial sensors and a new test battery for distinguishing retrospective fallers from non-fallers among community-dwelling older people. Sci Rep 2018 Nov 05;8(1):16349 [FREE Full text] [doi: 10.1038/s41598-018-34671-6] [Medline: 30397282]

77. Hsieh KL, Roach KL, Wajda DA, Sosnoff JJ. Smartphone technology can measure postural stability and discriminate fall risk in older adults. Gait Posture 2019 Jan;67:160-165. [doi: 10.1016/j.gaitpost.2018.10.005] [Medline: 30340129]

78. Maneeprom N, Taneepanichskul S, Panza A, Suputtitada A. Effectiveness of robotics fall prevention program among elderly in senior housings, Bangkok, Thailand: a quasi-experimental study. Clin Interv Aging 2019;14:335-346. [doi:

10.2147/CIA.S182336] [Medline: 30863027]

79. Nightingale CJ, Mitchell SN, Butterfield SA. Validation of the Timed Up and Go Test for assessing balance variables in adults aged 65 and older. J Aging Phys Act 2019 Apr 01;27(2):230-233. [doi: 10.1123/japa.2018-0049] [Medline: 30117359] 
80. Viirre E. Vestibular telemedicine and rehabilitation. Applications for virtual reality. Stud Health Technol Inform 1996;29:299-305. [Medline: 10163763]

\author{
Abbreviations \\ ICT: information and communication technologies \\ JBI: Joanna Briggs Institute \\ QES: quasiexperimental study \\ RCT: randomized controlled trial \\ TUGT: Timed Up and Go Test

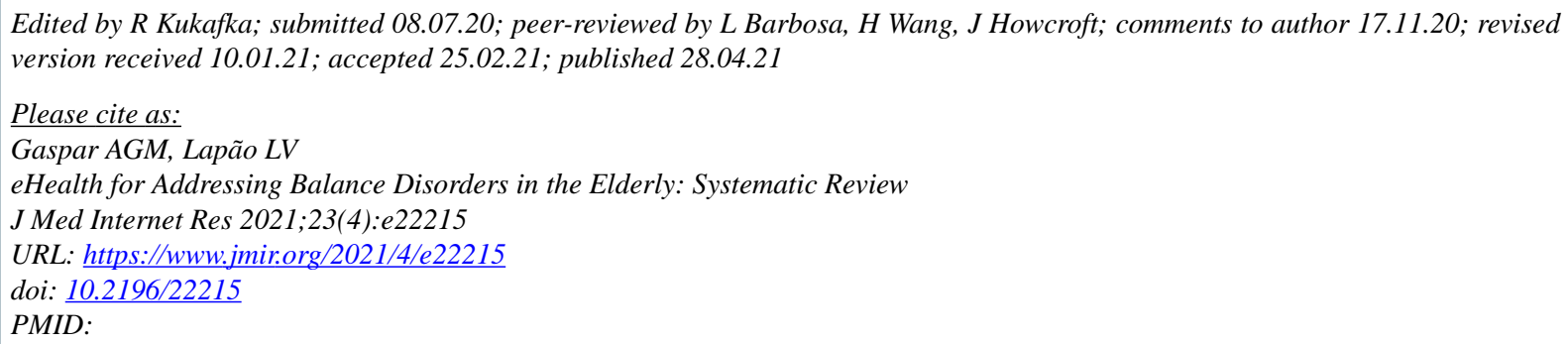

(C)Andréa G Martins Gaspar, Luís Velez Lapão. Originally published in the Journal of Medical Internet Research (https://www.jmir.org), 28.04.2021. This is an open-access article distributed under the terms of the Creative Commons Attribution License (https://creativecommons.org/licenses/by/4.0/), which permits unrestricted use, distribution, and reproduction in any medium, provided the original work, first published in the Journal of Medical Internet Research, is properly cited. The complete bibliographic information, a link to the original publication on http://www.jmir.org/, as well as this copyright and license information must be included. 